\title{
Theory and Applications of Numerical Simulation of Permeation Fluid Mechanics of the Polymer-Black Oil
}

\author{
Yirang Yuan ${ }^{1}$, Aijie Cheng ${ }^{1}$, Danping Yang ${ }^{1} \&$ Changfeng $\mathrm{Li}^{1,2}$ \\ ${ }^{1}$ Institute of Mathematics, Shandong University, Jinan, China \\ ${ }^{2}$ School of Economics, Shandong University, Jinan, China \\ Correspondence: Yirang Yuan, Institute of Mathematics, Shandong University, Jinan, China. Tel: \\ 86-531-88364732. E-mail: yryuan@sdu.edu.cn
}

\author{
Received: July 7, 2014 Accepted: August 212014 Online Published: October 24, 2014 \\ doi:10.5539/jgg.v6n4p12 URL: http://dx.doi.org/10.5539/jgg.v6n4p12
}

The research is financed by National climbing plan (Grant No: 1990328), National Tackling Key Project (Grant Nos: 2011ZX05011-004, 2011ZX05052, 20050200069), National Natural Science Foundation of China (Grant Nos: 11101244, 11271231, 10771124, 10372052), National Doctoral Foundation (Grant No: 20030422047) and Natural Science Foundation of Shandong Province (Grant No: ZR2011AM015).

\begin{abstract}
Theory and application of numerical simulation of permeation fluid mechanics is discussed about the polymer--black oil (water, oil and gas) in this paper. In view of petroleum geology, geochemistry, computational mechanics of flow and computer technology, the mechanics model of three-phase flow (water, oil and gas) of the polymer is presented firstly, then a numerical algorithm consisting of a full implicit program, and an implicit computation for the pressure and an explicit computation for the concentration is given by structuring an upstream sequence and an iterative algorithm of implicit fined upwind fractional step finite difference to solve the pressure equation, the concentration equation and the saturation of chemical substance components. A type of software applicable in major industries has been made in consideration of ten-meters steps, hundreds of thousands nodes and tens of years and high accuracy and the application has been carried out successfully in analysis and simulation of major oil-fields extraction such as Daqing Oilfield, Shengli Oilfield and Dagang Oilfield and others, which gives rise to outstanding economic and social benefits. A precise analysis is given for a simplified model and an idea is presented to solve this international famous problem.
\end{abstract}

Keywords: polymer flooding, mechanics of three-phase flow (water, oil and gas) through porous media - the polymer, model and numerical simulation, application of oil fields, theoretical analysis

\section{Introduction}

At present an effective method, water-flooding, to hold the pressure of reservoirs is popular in the world, and the recovery efficiency is more outstanding than any other natural exploring forms. It gives more benefits and helps Chinese oil fields keep high quantity production. It continues to be more important and how a strategic project works to develop the exploiting efficiency of crude oil in the way of water-flooding driving.

A mass of residual crude oil remains in the reservoir after water-flooding exploiting because of the constraint of capillary force preventing the motion and the slight influenced regions and the fluidity ratio between displacement phase and driven phase weakening the displacement force. Then it is more important to develop the displacement efficiency. A popular method is considered that the mixture is injected into the underground fluid including chemical addition agents such as polymer, surface active agent and alkali. The polymer can optimize the fluidity of displacement phase, modify the ratio with respect to driven phases, balance the leading edges well, weaken the inner porous layer, increase the efficiency of displacement and the pressure gradient. Surface active agent and alkali can decrease interfacial tensions of different phases, then make the bound oil move and gather.

Some hypotheses should be made as follows to find the mathematical models. The mixture fluid flows along isothermal curves, different phases keep equilibrium state, different components have no chemical reaction and expanded Darcy Theory holds and so on. In view of the pressure $p(x, t)$ and the saturation $c_{i}(x, t)$, the flow equation and convection-diffusion equations are derived with corresponding boundary value conditions and 
initial conditions.

The equilibrium equation of multi-phase, multi-components and slight compressible mixture is formulated by a nonlinear coupled partial differential equations. It is hard to solve this system because many modern numerical methods such as mixed element, finite element, finite difference and numerical algebra, will be involved in the simulation. In general speaking, based on physical means the pressure function is solved by an implicit scheme and the concentration values are obtained by an explicit solver or an implicit solver. The scholars try to find good ways analyzing the data and numerical results and doing some research work in simulation, which describe the whole process of chemistry displacements very well and help the engineers control the rules and process of displacement and forecast the recovery efficiency of natural oil and compute the oil percentage of output liquid and the percent of polymer and surface active agent. By numerical research the curves describing different components motion are shown, and some plans are made about the beginning and end of injected liquid and some related parameters of natural oil efficiency are derived. These conclusions, important techniques in chemistry displacements, can used in forecasting the characters of fields, choosing different optimization plans, establishing the models of chemical displacements of reservoir, completing computational software and carrying out the numerical simulation. Petroleum engineers and mathematicians pay more attention to modern new techniques of exploiting natural oil.

Yuan visited United States and accomplished some work cooperate with Prof. R. E. Ewing during 1985 to 1988 , and kept a series of research in theoretical analysis and applications of numerical simulation. Yuan and his research group $(1993,1993,1994)$ undertake some important projects from 1991 to 1995 such as "Eighth-Five" national key science and technology program (the Program for Tackling Key Programs) (85-203- 01-087) entitled "research and application of the polymer displacement software" (Note 1). The software has been applied in designing plan and research work of polymer displacements in industrial production region of Daqing Oilfield. Many conclusions from actual numerical results are illustrated by Yuan, Yang and Qi $(1998,2000)$ such as effects of fragments, fragments setting of rinsing protection, quantity of polymer, and used in actual simulations which give rise to outstanding economic and social benefits (Note 2). Later the authors undertook a key tackling program of oil administration of Daqing Oilfield (DQYJ-1201002-2006-JS-9565)----solving development of mathematical models and completing explain of reservoir (Note 3). This software system is also applied in numerical simulation of the polymer displacement of Zaobei fault block of Dagang Oilfield, optimization of designing plan of expanded experimental area of three compound combination flooding of Gudong Little Well experimental region of Shengli Oilfield, polymer flooding of Gudong Middle One experimental region, Gudong West region and feasibility of active water flooding of Gudong eighth region, and many interesting results are obtained (Note 4).

This paper concludes the former research and discusses proceeding analysis, mainly consisting of mathematical models of numerical simulation, numerical methods, applicable software, theoretical analysis and applications of mechanical polymer displacement of porous media.

\section{Permeation Fluid Mechanical Models of Black Oil-Polymer Displacement}

The numerical simulation program of black oil model is accomplished in view of the following factors: three phases (oil, gas and water) in the reservoir, oil-component and solution gas-component of the oil phase, water-component of the water phase, gas-component of the gas phase and the exchange of gas-component between the oil phase and the gas phase because of the changing pressure. An improved black-oil model is given to simulate the displacement of the polymer after considering the black oil module and the polymer module, and a new simulation system is made applied in actual oil-fields.

The oil, water and gas three phases are considered in the system, where the oil phase consists of oil component and solution gas component, the water phase includes water component, polymer component and anion, kation components, and the gas phase only has gas component. The exchange of gas components occurs between the oil phase and the gas phase because of the pressure of neighbor environments. The solving system consists of two basic modules: the program for solving the three-phase flow and the program for solving the component equations.

The solving module of three phases inherits a partial algorithm of the black oil model. The water viscosity is a variable in the polymer displacement model but a constant in the black oil model. Additional design structure and algorithm should be compatible with the black oil model for solving the component equation, and some computational programs are modified for engineering applications of wedge-out area, fault and edge-bottom water.

Then two different algorithms are presented in this paper. 
(I) Full implicit algorithm (FIA). It is one of the most dependable finite differences to compute the values of variables implicitly such as the pressure, the water saturation, the gas saturation and the ratio of solution gas and oil. Most time is taken for a full implicit scheme to complete each extrapolation iterative. The full implicit scheme proceeds more stable than an implicit/explicit algorithm for the pressure and saturation (IEAFPS) invalid sometimes because of strong stability conditions, so a large time step is introduced to decrease the total simulation cost.

(II) Implicit/explicit algorithm for the pressure and saturation (IEAFPS). This algorithm consists of an implicit scheme for solving the pressure and an explicit scheme for solving the saturation based on the following hypothesis: no distinct flow of the fluid occurs though the saturation changes during one time step. This hypothesis can cancel the unknown saturation of discretized convection equation. The values of saturation is updated point by point explicitly when the pressure changes keep implicit coupling and the change rules are determined during one iteration process. While the IEAFPS is unstable as the saturation changes faster with respect to time step. This implicit/explicit algorithm is very efficient as the time step is taken sufficiently small to decrease the rangeability (the relative rangeability usually be $5 \%$ ).

Let " $w "$ and " $o$ " denote the water phase and the oil phase of water-oil two phases problem, whose mathematical model of permeation fluid mechanics is stated as follows (see Ewing, Yuan, \& Li, 1989; Yuan, 2013; Yuan, Yang, \& Qi, 1998; Yuan, 2000):

$$
\begin{gathered}
\frac{\partial}{\partial x}\left[\lambda_{l}\left(\frac{\partial p_{l}}{\partial x}-\gamma_{l} \frac{\partial z}{\partial x}\right)\right]=\frac{\partial}{\partial t}\left(\phi \frac{\partial S_{l}}{\partial B_{l}}\right)-q_{l}, l=w, o ; \\
p_{c}=p_{o}-p_{w}, S_{o}+S_{w}=1.0 .
\end{gathered}
$$

Let " $w ", " o "$ and " $g$ " respectively denote the water phase, the oil phase and the gas phase, and the mathematical model of three phases permeation fluid mechanics is followed ${ }^{[12(3)(4)}$,

$$
\left\{\begin{array}{c}
\frac{\partial}{\partial x}\left[\lambda_{w}\left(\frac{\partial p_{w}}{\partial x}-\gamma_{w} \frac{\partial z}{\partial x}\right)\right]=\frac{\partial}{\partial t}\left(\phi \frac{\partial S_{w}}{\partial B_{w}}\right)-q_{w}, \\
\frac{\partial}{\partial x}\left[\lambda_{o}\left(\frac{\partial p_{o}}{\partial x}-\gamma_{o} \frac{\partial z}{\partial x}\right)\right]=\frac{\partial}{\partial t}\left(\phi \frac{\partial S_{o}}{\partial B_{o}}\right)-q_{o}, \\
\frac{\partial}{\partial x}\left[R_{s} \lambda_{o}\left(\frac{\partial p_{o}}{\partial x}-\gamma_{o} \frac{\partial z}{\partial x}\right)\right]+\frac{\partial}{\partial x}\left[\lambda_{g}\left(\frac{\partial p_{g}}{\partial x}-\gamma_{g} \frac{\partial z}{\partial x}\right)\right]=\frac{\partial}{\partial t}\left(\phi R_{s} \frac{1-S_{w}-S_{g}}{\partial B_{o}}+\phi \frac{\partial S_{g}}{\partial B_{o}}\right)-R_{s} q_{o}-q_{g} ; \\
p_{o}-p_{w}=p_{c o w}, p_{g}-p_{o}=p_{c o g}, \\
\lambda_{l}=\frac{K K_{r l}}{\mu_{l} B_{l}}, l=o, w, g, \gamma_{l}=\rho_{l} g, \\
\mathrm{u}_{l}=\lambda_{l}\left(\nabla p_{l}-\gamma_{l} \nabla z\right), l=w, o, g .
\end{array}\right.
$$

where $\phi$ means the porosity, $p_{l}$ is the pressure of $l$-phase, $S_{l}$ is the concentration of $l$-phase, $K$ is the absolute permeability, $B_{l}$ is the volume factor of $l$-phase, $K_{r l}$ is the relative permeability of $l$-phase, $\mu_{l}$ is the viscosity of $l$-phase, $\rho_{l}$ is the density of $l$-phase, $R_{s}$ is the ratio of solution gas and oil, and $q_{l}$ is the source sink term of $l$-phase (floor condition).

The viscosity of water phase $\mu_{w}$ is a constant of black oil model, while the viscosity is a function with respect to the density of polymer of black oil-polymer displacement, $\mu_{w}=\mu_{w}\left(C_{p w}\right)$, where $C_{p w}$ denotes the concentration of polymer relative to water. The polymer components motion in water, the concentration affects in turn the viscosity field of the water phase, then influence the flow of the three phase fluid accompanying the motion of components. The coupled algorithm runs consistent with the nonlinear coupled system concluding a convection-diffusion equation of the polymer and the mathematical model of black oil. At a time step the motion values of three phases are computed first, the flow field is obtained, then the solution of convection diffusion equation are gotten, then the concentration field of polymer and the viscosity field of water phase are updated. Then the computation proceeds at the next time step.

The following convection diffusion equation explains the motion law of the polymer, anions and cations, for convenience the different components are not distinguished and the subscript $w$ denotes the concentration of a certain component.

$$
\frac{\partial}{\partial t}\left(\phi S_{w} C\right)+\operatorname{div}\left(C \mathrm{u}_{w}-\phi S_{w} K \nabla C\right)=Q
$$




$$
\mu_{w}=\mu_{w}(C) .
$$

Eqs. (1a), (1b), (2e) and (2f) give a full description of the mathematical model of water and oil two-phase polymer displacement, and eqs. (2a), (2b), (2c), (2d), (2e) and (2f) represent the full explanation of the mathematical model of water-oil-gas three phases polymer displacement.

The computation of the black oil-polymer displacement model runs in the following steps:

Step 1. At the time level $t^{1}$, solve the pressure and saturation, then solve the concentration of the components.

Step 2. Correct the viscosity of water phase dependent on the concentration.

Step 3. At the time level $t^{2}$, solve the pressure and saturation, then solve the concentration of the components

Step 4. Correct the viscosity of water phase dependent on the concentration.

......

In a similar way the pressure and saturation of the $(n+1)$ th time step $t^{n+1}$ are obtained, the concentration of the components at $t^{n+1}$ is computed, then the viscosity of water phase is corrected according to the concentration.

The program ends.

\section{Numerical Computation Method}

Two different numerical methods are presented as follows.

\subsection{Full Implicit Numerical Method of the Three Phases Flow}

It aims to compute three unknown variables, generally the pressure of oil phase, the concentrations of water and gas phases are considered and other spare unknown numbers are cancelled. All the values of the left side of the equation such as the pressure, the saturation, the quantity, the relative permeability, the capillary force and other parameters are taken the latest numerical values during implicit computations. The resulting implicit difference is a nonlinear algebraic equations solved by iteration and its computation scale is more rich seven times the implicit/explicit method one iteration (outer iteration). By that the full implicit method is unconditionally stable, it is applied to compute some difficult and complicated problems such as black oil simulation. In this paper let $\bar{\delta}$ denote the values after one time step and let $\delta$ be the values of the $(k+1)$-th iteration after one iteration.

$$
\bar{\delta} f=f^{n+1}-f^{n}, \quad \delta f=f^{k+1}-f^{k}, \bar{\delta} f \approx f^{k+1}-f^{n}=f^{k}+\delta f-f^{n} .
$$

Applying Euler backwards finite difference method for eq. (2),

$$
\begin{aligned}
& \left\{\left(\Delta T_{l} \Delta\left(p_{l}-\gamma_{l} z\right)\right)^{n+1}+\omega\left[\Delta\left(T_{o} R_{s} \Delta\left(p_{o}-\gamma_{o} z\right)\right)^{n+1}+\left(q_{o} R_{s}\right)^{n+1}\right]\right\}_{i} \\
& =\frac{V_{b}}{\Delta t}\left\{\left(\phi \frac{S_{l}}{B_{l}}\right)+\omega\left(\frac{1}{B_{o}} R_{s} S_{o}\right)\right\}_{i}^{n+1}-q_{l i}^{n+1}, l=w, o, g,
\end{aligned}
$$

where $\omega=1$ as $l=g$ and $\omega=0$ as $l=w, o$, and $V_{b}=\Delta x \Delta y \Delta z$.

Then a full implicit finite difference method for the black oil model is derived as follows

$$
\Delta T_{l}^{n+1} \Delta \Phi_{l}^{n+1}+q_{l}^{n+1}+\omega\left[\Delta\left(T_{o} R_{s} \Delta \Phi_{o}\right)^{n+1}+\left(q_{o} R_{s}\right)^{n+1}\right]=\frac{V_{b}}{\Delta t} \bar{\delta}\left[\phi b_{l} S_{l}+\omega\left(b_{o} R_{s} S_{o}\right)\right], l=w, o, g,
$$

where $b_{l}=1 / B_{l}$ and $\Phi=p_{i}-\gamma_{l} D$. Rewrite the operator $\delta$ as follows,

$$
\begin{aligned}
& \Delta\left(T_{l}^{k}+\delta T_{l}\right) \Delta\left(\Phi_{l}^{k}+\delta \Phi_{l}\right)+q_{l}^{k}+\delta q_{l}+\omega\left\{\left[\Delta\left(T_{o} R_{s}\right)^{k}+\delta\left(T_{o} R_{s}\right)\right]\left[\Delta\left(\Phi_{o}^{k}+\delta \Phi_{o}\right)+\left(q_{o} R_{s}\right)^{k}+\delta\left(q_{o} R_{s}\right)\right]\right\} \\
& =\frac{V_{b}}{\Delta t}\left\{\left[\phi b_{l} S_{l}+\omega\left(b_{o} R_{s} S_{o}\right)\right]^{k}+\delta\left[\phi b_{l} S_{l}+\omega\left(\phi b_{o} R_{s} S_{o}\right)\right]-\left[\phi b_{l} S_{l}+\omega\left(\phi b_{o} R_{s} S_{o}\right)\right]^{n}\right\}, l=w, o, g .
\end{aligned}
$$

Give an expansion of the above equation and omit quadratic terms, and the remainder after the $k$-th iteration is

$$
\begin{aligned}
R_{l}^{k} \equiv & \Delta T_{l}^{k} \Delta \Phi_{l}^{k}+q_{l}^{k}+\omega\left[\Delta\left(T_{o} R_{s}\right)^{k} \Delta \Phi_{o}^{k}+\left(q_{o} R_{s}\right)^{k}\right] \\
& -\frac{V_{b}}{\Delta t}\left\{\left[\phi b_{l} S_{l}+\omega\left(b_{o} R_{s} S_{o}\right)\right]^{k}-\left[\phi b_{l} S_{l}+\omega\left(\phi b_{o} R_{s} S_{o}\right)\right]^{n}\right\}, l=w, o, g .
\end{aligned}
$$

Continue to give an expression with remainder term, 


$$
\begin{aligned}
& \Delta\left(\delta T_{l}\right) \Delta \Phi_{l}^{k}+\Delta T_{l}^{k} \Delta\left(\delta \Phi_{l}\right)+\delta q_{l}+\omega\left[\Delta \delta\left(T_{o} R_{s}\right) \Delta \Phi_{o}^{k}+\Delta\left(T_{o} R_{s}\right)^{k} \Delta\left(\delta \Phi_{o}\right)+\delta\left(q_{o} R_{s}\right)\right] \\
& =\frac{V_{b}}{\Delta t} \delta\left[\phi b_{l} S_{l}+\omega\left(\phi b_{o} R_{s} S_{o}\right)\right]-R_{l}^{k} .
\end{aligned}
$$

The iterations are convergent as $R_{l}^{k} \rightarrow 0$ for $l=w, o, g$ and $k=1,2, \ldots$ A resulting formulation is

$$
R H S_{l}=C_{l 1} \delta p_{o}+C_{l 2} \delta S_{w}+C_{l 3} \delta S_{g}-R_{l}^{k}, l=w, o, g .
$$

First, the equation is turned into a linearized expansion. Solving variables are denoted by $\delta p_{o}, \delta S_{w}$ and $\delta S_{g}$, and the right side term is considered later.

Notice that $\delta(a b)=a^{k+1} \delta b+b^{k} \delta a$ and consider the right side term of water phase,

where

$$
\begin{gathered}
R H S_{w}=\frac{V_{b}}{\Delta t} \delta\left(\phi b_{w} S_{w}\right)-R_{w}^{k}=C_{w 1} \delta p_{o}+C_{w 2} \delta S_{w}+C_{w 3} \delta S_{g}-R_{w}^{k}, \\
C_{w 1}=\frac{V_{b}}{\Delta t} S_{w}^{k}\left(\phi^{k+1} b_{w}+b_{w}^{k} \phi^{\prime}\right), C_{w 2}=\frac{V_{b}}{\Delta t} \phi^{k+1}\left(b_{w}^{k+1}-S^{k} b_{w}^{\prime} p_{c w o}^{\prime}\right), C_{w 3}=0, \\
R_{w}^{k}=\Delta T_{w}^{k} \Delta \Phi_{w}^{k}+q_{w}^{k}-\frac{V_{b}}{\Delta t}\left[\left(\phi b_{w} S_{w}\right)^{k}-\left(\phi b_{w} S_{w}\right)^{n}\right] .
\end{gathered}
$$

Consider the right side term of oil phase equation,

where

$$
\begin{aligned}
& R H S_{o}=\frac{V_{b}}{\Delta t} \delta\left(\phi b_{o} S_{o}\right)-R_{o}^{k}=C_{o 1} \delta p_{o}+C_{o 2} \delta S_{w}+C_{o 3} \delta S_{g}-R_{o}^{k}, \\
& C_{o 1}=\frac{V_{b}}{\Delta t} S_{o}^{k}\left(\phi^{k+1} b_{o}^{\prime}+b_{o}^{k} \phi^{\prime}\right), C_{o 2}=\frac{V_{b}}{\Delta t} \phi^{k+1}\left(\phi b_{o}\right)^{k+1}, C_{w 3}=-\frac{V_{b}}{\Delta t} \phi^{k+1}\left(\phi b_{o}\right)^{k+1}, \\
& R_{o}^{k}=\Delta T_{o}^{k} \Delta \Phi_{o}^{k}+q_{o}^{k}-\frac{V_{b}}{\Delta t}\left[\left(\phi b_{o} S_{o}\right)^{k}-\left(\phi b_{o} S_{o}\right)^{n}\right] .
\end{aligned}
$$

Consider the right side term of gas-phase equation,

where

$$
R H S_{g}=\frac{V_{b}}{\Delta t} \delta\left[\left(\phi b_{g} S_{g}\right)+\left(\phi b_{o} R_{s} S_{o}\right)\right]-R_{g}^{k}=C_{g 1} \delta p_{o}+C_{g 2} \delta S_{w}+C_{g 3} \delta S_{g}-R_{g}^{k}
$$

$$
\begin{aligned}
& C_{g 1}=\frac{V_{b}}{\Delta t}\left\{\left(b_{g} S_{g}+b_{o} R_{s} S_{o}\right)^{k} \phi_{r} C_{r}+\phi^{k+1}\left[S_{g}^{k} b_{g}^{\prime}+S_{o}^{n}\left(R_{s}^{k+1} b_{o}^{\prime}+b_{o}^{k} R_{s}^{\prime}\right)\right]\right\}, \\
& C_{g 2}=-\frac{V_{b}}{\Delta t}\left(\phi b_{o} R_{s}\right)^{k+1}, C_{g 3}=-\frac{V_{b}}{\Delta t} \phi^{k+1}\left(b_{g}^{k+1}+S_{g}^{k} b_{g}^{\prime} p_{c g o}^{\prime}\right)+C_{g 2}, \\
& R_{g}^{k}=\Delta T_{g}^{k} \Delta \Phi_{g}^{k}+q_{g}^{k}+\left[\Delta\left(T_{o} R_{s}\right)^{k} \Delta \Phi_{o}^{k}+\left(q_{o} R_{s}\right)^{k}\right]-\frac{V_{b}}{\Delta t}\left\{\left[\phi\left(b_{g} S_{g}+b_{o} R_{s} S_{o}\right)\right]^{k}-\left[\phi\left(b_{g} S_{g}+b_{o} R_{s} S_{o}\right)\right]^{n}\right\} .
\end{aligned}
$$

In the above expressions $b_{l}{ }^{\prime}$ and $\phi^{\prime}$ present the derivatives of volume factor and porosity with respect to the pressure, $p_{c o w}^{\prime}$ denotes the derivative of $p_{c}$ with respect to $S_{w}$ and $p_{c g o}^{\prime}$ is the derivative of $p_{c}$ with respect to $S_{g}$.

Two operators are introduced to discuss the left side term,

$$
M_{l}=\Delta T_{l}^{k} \Delta\left(\delta \Phi_{l}\right)^{k}+\omega\left[\Delta\left(T_{o} R_{s}\right)^{k} \Delta\left(\delta \Phi_{o}\right)\right], N_{l}=\Delta\left(\delta T_{l}\right) \Delta \Phi_{l}^{k}+\omega\left[\Delta \delta\left(T_{o} R_{s}\right) \Delta \Phi_{o}^{k}\right] .
$$

The difference equation is rewritten as follows,

$$
M_{l}+N_{l}+\delta q_{l}+\omega \delta\left(q_{o} R_{s}\right)=R H S_{l}, l=w, o, g .
$$

Here an expansion of $M_{l}$ is only considered. For water phase,

$$
M_{w}=\Delta T_{w}^{k} \Delta\left(\delta \Phi_{w}\right) \approx \Delta T_{w}^{k} \Delta(\delta p)-\Delta T_{w}^{k} \Delta\left(p_{c w o}^{\prime} \delta S_{w}\right),
$$

for oil phase, 


$$
M_{o}=\Delta T_{o}^{k} \Delta\left(\delta \Phi_{o}\right) \approx \Delta T_{o}^{k} \Delta\left[\delta\left(p-\gamma_{o} D\right)\right] \approx \Delta T_{w}^{k} \Delta(\delta p),
$$

and for gas phase,

$$
M_{g}=\Delta T_{g}^{k} \Delta\left(\delta \Phi_{g}\right)+\Delta\left(\mathrm{T}_{o} \mathrm{R}_{s}\right)^{k} \Delta\left(\delta \Phi_{o}\right) \approx \Delta\left(T_{g}+\mathrm{T}_{o} \mathrm{R}_{s}\right)^{k} \Delta(\delta p)-\Delta T_{g}^{k} \Delta\left(p_{c g o}^{\prime} \delta S_{g}\right) .
$$

The values of conductivity coefficient appearing in two-order difference operator are given according to upstream rule, and let $i_{+}$and $i_{-}$denote the upstream nodes between the $i$-th node and the $(i+1)$-th node and between the $i$-th node and the (i-1)-th node, respectively,

$$
\Delta T_{l} \Delta(\delta \mathrm{f})_{i}=T_{l i_{+}}\left(\delta f_{i+1}-\delta f_{i}\right)-T_{l i_{-}}\left(\delta f_{i}-\delta f_{i-1}\right), l=w, o, g .
$$

Substitute the left side and the right side terms into the initial difference equation, and get the algebraic system.

\subsection{Implicit/explicit Algorithm for the Pressure and the Saturation}

The implicit/explicit method is based on an equation only involved of the pressure by adding all the flow equations, and the values of the saturation are obtained explicitly as the pressure at a certain time level is known.

Discrete difference scheme of eq. (2) is written related with $p_{o}$ and the saturation,

$$
\begin{aligned}
& \Delta\left[T_{w}\left(\Delta p_{o}-\Delta p_{c o w}-\gamma_{w} \Delta z\right)\right]=C_{1 p} \Delta_{t} p_{w}+\sum_{t} C_{1 l} \Delta_{t} S_{l}+q_{w}, \Delta\left[T_{o}\left(\Delta p_{o}-\gamma_{o} \Delta z\right)\right]=C_{2 p} \Delta_{t} p_{o}+\sum_{t} C_{2 l} \Delta_{t} S_{l}+q_{o}, \\
& \Delta\left[T_{g}\left(\Delta p_{o}-\Delta p_{c o g}-\gamma_{g} \Delta z\right)\right]+\Delta\left[R_{s} T_{o}\left(\Delta p_{o}-\gamma_{o} \Delta z\right)\right]=C_{3 p} \Delta_{t} p_{g}+\sum_{t} C_{3 l} \Delta_{t} S_{l}+R_{s} q_{o}+q_{g} .
\end{aligned}
$$

A basic hypothesis is assumed for deriving the implicit/explicit scheme that the capillary pressure of the flow term of the left side is a constant number during a time step computation. The values of some terms related of $\Delta p_{\text {cow }}$ and $\Delta p_{\text {cog }}$ at the previous time level can be computed explicitly, and $\Delta_{t} p_{w}=\Delta_{t} p_{o}=\Delta_{t} p_{g}$. The notation $p_{o}$ is simplified to be replaced by $p$.

$$
\begin{aligned}
& \Delta\left[T_{w}\left(\Delta p^{n+1}-\Delta p_{c o w}^{n}-\gamma_{w} \Delta z\right)\right]=C_{1 p} \Delta_{t} p+C_{1 w} \Delta_{t} S_{w}+q_{w}, \Delta\left[T_{o}\left(\Delta p^{n+1}-\gamma_{o} \Delta z\right)\right]=C_{2 p} \Delta_{t} p+C_{1 o} \Delta_{t} S_{o}+q_{o}, \\
& \Delta\left[T_{g}\left(\Delta p^{n+1}-\Delta p_{c o g}^{n}-\gamma_{g} \Delta z\right)\right]+\Delta\left[R_{s} T_{o}\left(\Delta p^{n+1}-\gamma_{o} \Delta z\right)\right]=C_{3 p} \Delta_{t} p+C_{3 o} \Delta_{t} S_{o}+C_{3 g} \Delta_{t} S_{g}+R_{s} q_{o}+q_{g} .
\end{aligned}
$$

The coefficient $C$ is defined by

$$
\begin{aligned}
& C_{1 p}=\frac{V_{b}}{\Delta t}\left[\left(S_{w} \phi\right)^{n} b_{w}^{\prime}+S_{w}^{n} n_{w}^{n+1} \phi^{\prime}\right], C_{1 w}=\frac{V_{b}}{\Delta t}\left(\phi b_{w}\right)^{n+1}, \\
& C_{2 p}=\frac{V_{b}}{\Delta t}\left[\left(S_{o} \phi\right)^{n} b_{o}^{\prime}+S_{o}^{n} b_{o}^{n+1} \phi^{\prime}\right], C_{2 o}=\frac{V_{b}}{\Delta t}\left(\phi b_{o}\right)^{n+1}, \\
& C_{3 p}=\frac{V_{b}}{\Delta t}\left[R_{s}^{n}\left(S_{o}^{n} \phi^{n} b_{o}^{\prime}+S_{o}^{n} b_{o}^{n+1} \phi^{\prime}\right)+S_{g}^{n} \Phi^{n} b_{g}^{\prime}+S_{g}^{n} b_{g}^{n+1} \phi^{\prime}+\left(\phi S_{o} b_{o}\right)^{n+1} R_{s}^{\prime}\right], \\
& C_{3 o}=\frac{V_{b}}{\Delta t}\left[R_{s}^{n}\left(\phi b_{o}\right)^{n+1}\right], C_{3 g}=\frac{V_{b}}{\Delta t}\left(\phi b_{g}\right)^{n+1} .
\end{aligned}
$$

Consider the three equations of (11) together and cancel all the $\Delta_{t} S_{l}$ terms by multiplying the water phase equation by $A$, testing the gas equation by $B$, and adding three equations. The right term is

$$
\left(A C_{1 p}+C_{2 p}+B C_{3 p}\right) \Delta_{t} p+\left(-A C_{1 w}+C_{2 o}+B C_{3 o}\right) \Delta_{t} S_{o}+\left(-A C_{1 w}+B C_{3 p}\right) \Delta_{t} S_{g} .
$$

The numbers $A$ and $B$ are defined by the following relations.

$$
-A C_{1 w}+C_{2 o}+B C_{3 o}=0, \quad-A C_{1 w}+B C_{3 g}=0 .
$$

By simple algebraic calculations,

$$
B=C_{2 o} /\left(C_{3 g}-C_{3 o}\right), \quad A=B C_{3 g} / C_{1 w} .
$$

Then the pressure equation turns into 


$$
\begin{aligned}
& \Delta\left[T_{o}\left(\Delta p^{n+1}-\gamma_{o} \Delta z\right)\right]+A \Delta\left[T_{w}\left(\Delta p^{n+1}-\gamma_{w} \Delta z\right)\right]+B \Delta\left[T_{o} R_{s}\left(\Delta p^{n+1}-\gamma_{o} \Delta z\right)+T_{g}\left(\Delta p^{n+1}-\gamma_{g} \Delta z\right)\right] \\
& =\left(C_{2 p}+A C_{1 p}+B C_{3 p}\right) \Delta_{t} p+A \Delta\left(T_{w} \Delta p_{c o w}^{n}\right)-B \Delta\left(T_{w} \Delta p_{c o g}^{n}\right)+q_{o}+A q_{w}+B\left(R_{s} q_{o}+q_{g}\right) .
\end{aligned}
$$

It is a typical finite difference equation from parabolic type, whose matrix form is

$$
T p^{n+1}=D\left(p^{n+1}-p^{n}\right)+G+Q,
$$

where $T$ denotes a tridiagonal matrix and $D$ is a diagonal matrix. The vector $G$ is dependent of gravity and capillary pressure.

Given the pressure, taken in the former two equations of (11), the saturation $S_{l}^{n+1}$ is obtained explicitly. Then the capillary pressure $p_{c o w}^{n+1}$ and $p_{c o g}^{n+1}$ are considered, which are used explicitly in next time level.

\subsection{Numerical method for component concentration}

The components in water phase such as anions, cations, polymer molecules and so on are considered in this paper. The physical nature, conversation of mass, is described by a convection-diffusion equation but convection-dominated. It is more efficient and is of high order accuracy to decompose the equation into a hyperbolic equation only related of diffusion and a parabolic equation only related of dispersion. The former is discretized by an implicit upwind scheme and an upstream rule inheriting some advantages of explicit algorithms such as solving the values point by point. The latter is solved by an alternating direction finite difference method, which can accelerate the computation speed and improve the efficiency. For simplicity, the concentration equation of components is rewritten as a typical convection-diffusion expression

$$
\frac{\partial}{\partial t}\left(\phi S_{w} C\right)+\operatorname{div}\left(C \mathrm{u}_{w}-\phi S_{w} K \nabla C\right)=Q,
$$

where dispersion tensor is a matrix of diagonal type. When the saturation $S_{w}$ and the flow velocity field $\boldsymbol{u}_{w}$ of water phase at $t^{n+1}$ are given, it is to compute the concentration $C^{n+1}$ at the first direction, the second and then the third direction alternatively. $C$ denotes the concentration of any component ignoring the subscript $k$. A convection equation is discussed by an implicit upwind scheme,

$$
\begin{aligned}
& \frac{\phi_{i j k}^{n+1} S_{w}^{n+1} C_{i j k}^{n+1,0}-\phi_{i j k}^{n} S_{w}^{n} C_{i j k}^{n}}{\Delta t}+\frac{C_{i_{+}+j k}^{n+1,0} u_{w, i j k}^{n+1}-C_{i-j k}^{n+1,0} u_{w, i-1, j k}^{n+1}}{\Delta x}+\frac{C_{i j_{+} k}^{n+1,0} u_{w, i j k}^{n+1}-C_{i j-k}^{n+1,0} u_{w, i, j-1, k}^{n+1}}{\Delta y} \\
& +\frac{C_{i j k_{+}}^{n+1,0} u_{w, i j k}^{n+1}-C_{i j k_{-}}^{n+1,0} u_{w, i j, k-1}^{n+1}}{\Delta z}=Q_{i j k}^{n+1},
\end{aligned}
$$

by which $C^{n+1,0}$ is obtained. Then the dispersion equation is discussed alternatively in three directions. First consider $x$-direction,

$$
\begin{aligned}
& \frac{\phi_{i j k}^{n+1} S_{w}^{n+1} C_{i j k}^{n+1,1}-\phi_{i j k}^{n} S_{w}^{n} C_{i j k}^{n+1,0}}{\Delta t} \\
& +\frac{1}{(\Delta x)^{2}}\left\{\phi_{i+1 / 2, j k} S_{w, i_{+} j k}^{n+1} K_{x x, i+1 / 2, j k}\left(C_{i+1, j k}^{n+1,1}-C_{i j k}^{n+1,1}\right)-\phi_{i-1 / 2, j k} S_{w, i, j k}^{n+1} K_{x x, i-1 / 2, j k}\left(C_{i j k}^{n+1,1}-C_{i-1, j k}^{n+1,1}\right)\right\}=0 .
\end{aligned}
$$

Secondly consider $y$-direction,

$$
\begin{aligned}
& \frac{\phi_{i j k}^{n+1} S_{w}^{n+1} C_{i j k}^{n+1,2}-\phi_{i j k}^{n} S_{w}^{n} C_{i j k}^{n+1,1}}{\Delta t} \\
& +\frac{1}{(\Delta y)^{2}}\left\{\phi_{i, j+1 / 2, k} S_{w, i j_{+} k}^{n+1} K_{x x, i, j+1 / 2, k}\left(C_{i, j+1, k}^{n+1,1}-C_{i j k}^{n+1,1}\right)-\phi_{i, j-1 / 2, k} S_{w, i j-k}^{n+1} K_{x x, i, j-1 / 2, k}\left(C_{i j k}^{n+1,1}-C_{i, j-1, k}^{n+1,1}\right)\right\}=0 .
\end{aligned}
$$

Thirdly consider $z$-direction, then $C^{n+1}$ is computed by

$$
\begin{aligned}
& \frac{\phi_{i j k}^{n+1} S_{w}^{n+1} C_{i j k}^{n+1}-\phi_{i j k}^{n} S_{w}^{n} C_{i j k}^{n+1,2}}{\Delta t} \\
& +\frac{1}{(\Delta z)^{2}}\left\{\phi_{i j, k+1 / 2} S_{w, i j k_{+}}^{n+1} K_{x x, i j, k+1 / 2}\left(C_{i j, k+1}^{n+1,1}-C_{i j k}^{n+1,1}\right)-\phi_{i j, k-1 / 2} S_{w, i j k_{-}}^{n+1} K_{x x, i j, k-1 / 2}\left(C_{i j k}^{n+1,1}-C_{i j, k-1}^{n+1,1}\right)\right\}=0 .
\end{aligned}
$$

Now $p_{o}^{n+1}, S_{w}^{n+1}, S_{g}^{n+1}$ and $C^{n+1}$ at $t^{n+1}$ are known, and the computation continues at the next time level. 


\section{Computation Program Illustration}

This section illustrates three computation programs (Note 1, Note2, and Note 3): the program of black oil and polymer flooding, the data program of upstream sequence algorithm connected by faults, and the implicit computational program of the concentration equation of black oil and polymer components (see Fig.1, Fig.2 and Figure 3).

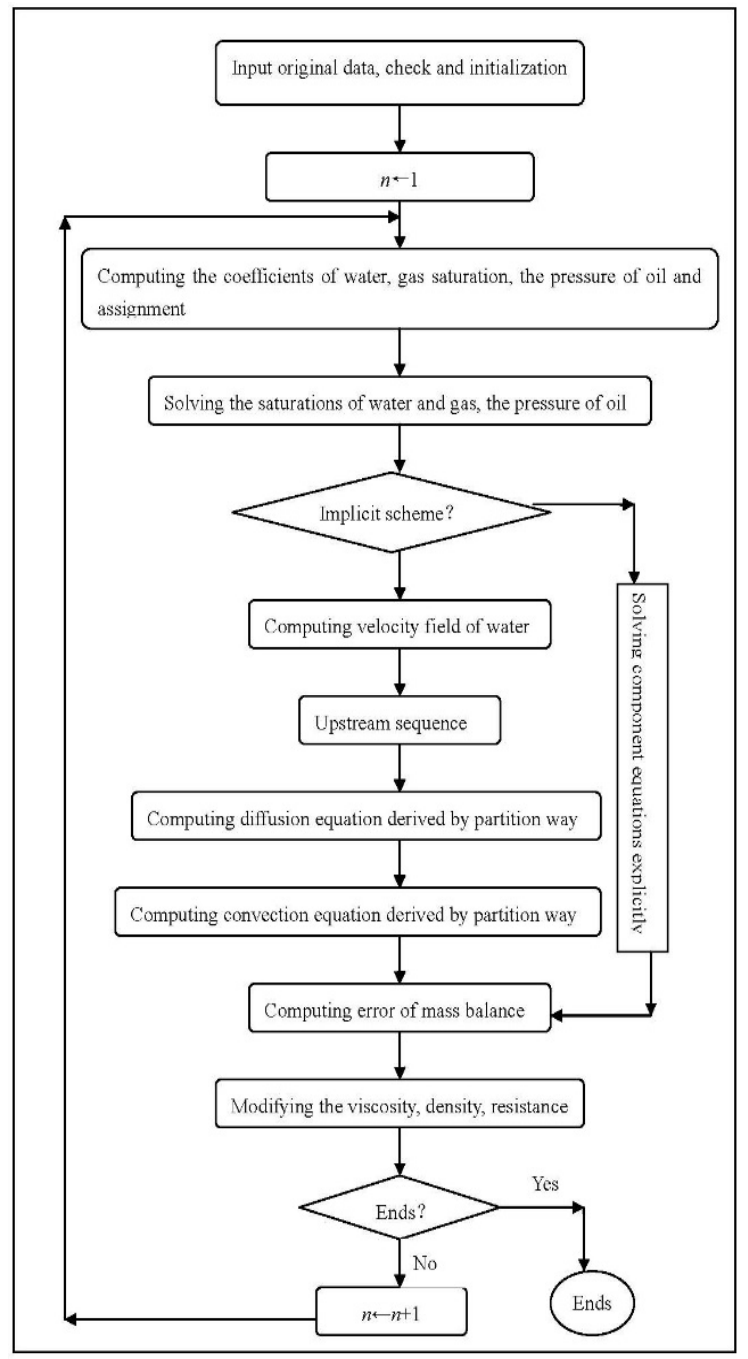

Fig. 1 Computation program of black oil and polymer flooding

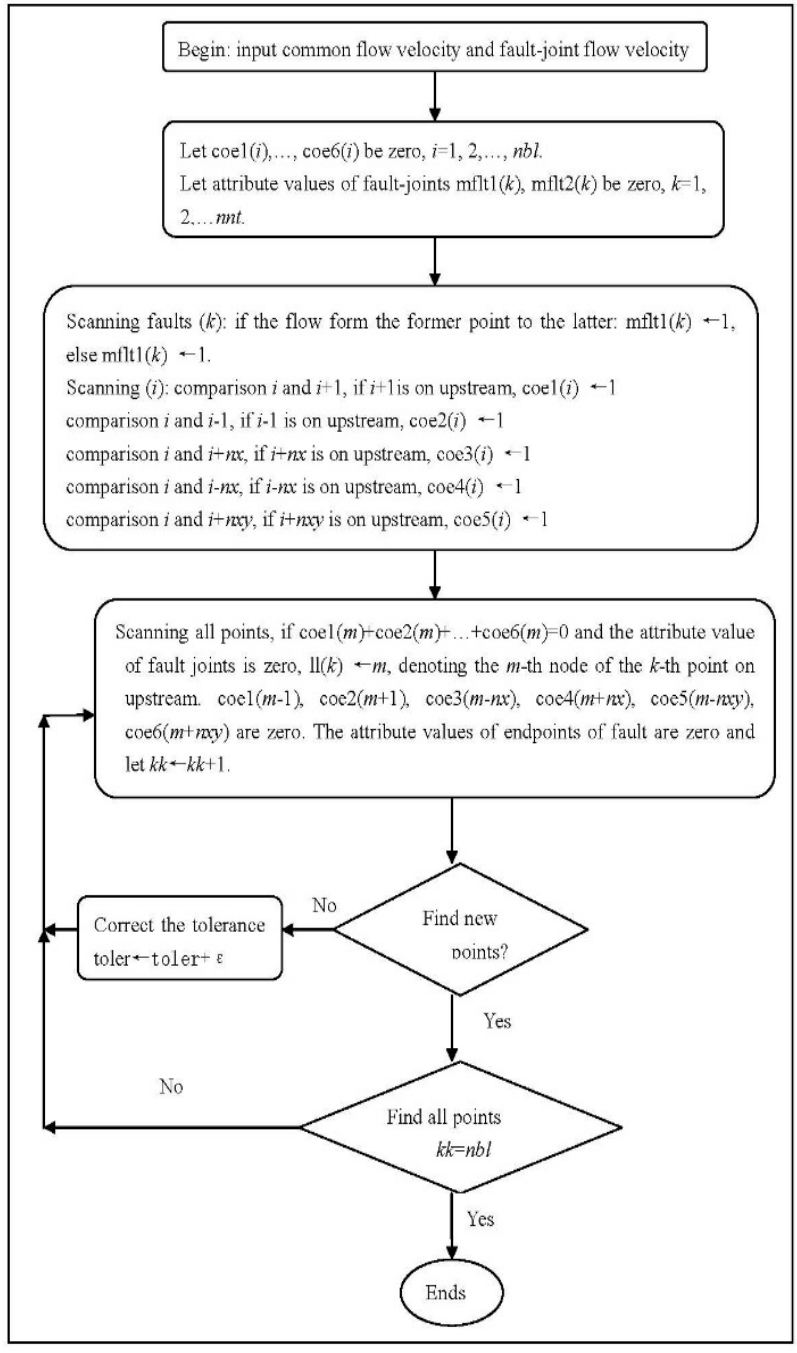

Fig. 2 Data program of upstream sequence of fault-joint points

\section{Experimental Tests for Black Oil and Polymer Flooding}

The model grids scale of experimental tests is defined by $9 \times 9 \times 3$, and its lengths in $x$-direction and $y$-direction are $D X=D Y=44.5$ meters and its height is $D Z=2.0$ meters. The simulation begins at January 1, 2000 and ends at January 1, 2015. The polymer is injected from January 1, 2004 to January 1, 2008, and the concentration is 2000 $\mathrm{mg} / \mathrm{L}$. Concentration isogram of the polymer, saturation isogram of water and viscosity isogram of water phase are illustrated in nine figures (Fig. 4-Fig. 6) in three different layers at the end of injection, January 1, 2008. These numerical data are shown in Fig.7, Fig. 8 and Fig. 9 layer by layer when the moisture content is $98 \%$ and the date is August 20,2011. During the whole injection and production, the moisture content of production wells, the curve of water yield and the curve of oil production are shown in Fig. $10^{(12)(3)}$. What numerical data conclude is that black oil and polymer flooding can precisely explain the physical process and mechanism of the displacement because the distribution curves of main physical quantities such as saturation, component concentration, viscosity of water and so on are reasonable, the order of accuracy is satisfactory, and undesired results such as polymer stacking and infinite circulation appear. Error estimates of mass balance of water, oil, gas, the polymer, anions and cations are respectively denoted by $2.71 E-6,2.70 E-7,2.70 E-7,1.58 E-15,7.84 E-16$, $2.00 E-15$. 


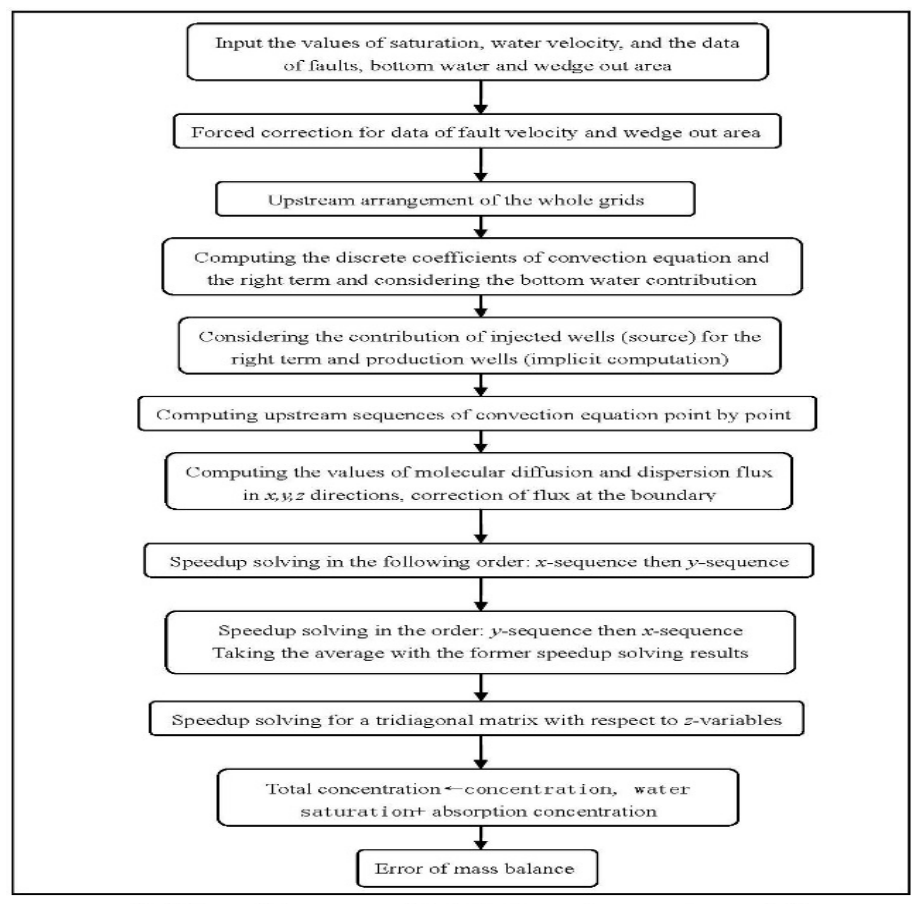

Fig. 3 Computation program of implicit scheme of component conecntration equation of black oil and polymer flooding

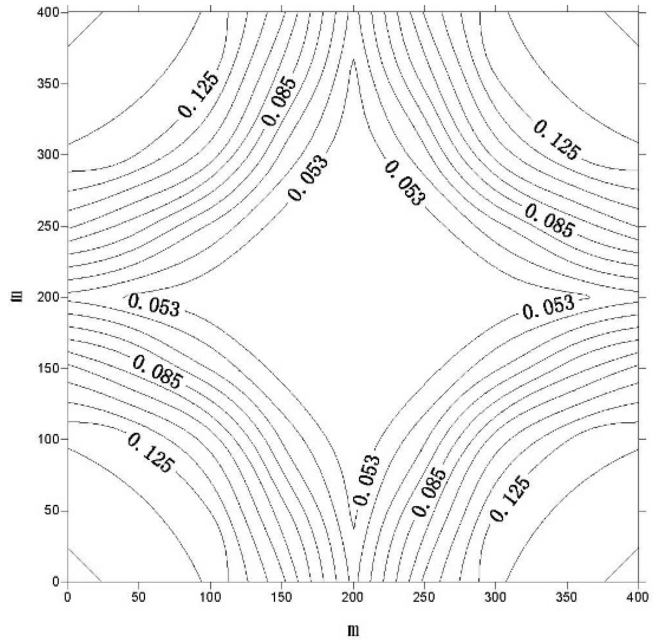

Fig. 4a Isogram of the polymer concentration in the first layer, date: $1 / 1 / 2008$

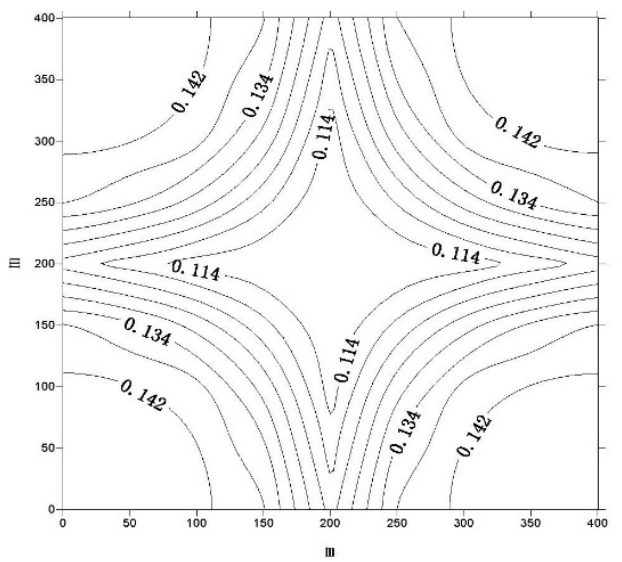

Fig. $4 \mathrm{c}$ Isogram of the polymer concentration in the third layer, date: 1/1/2008

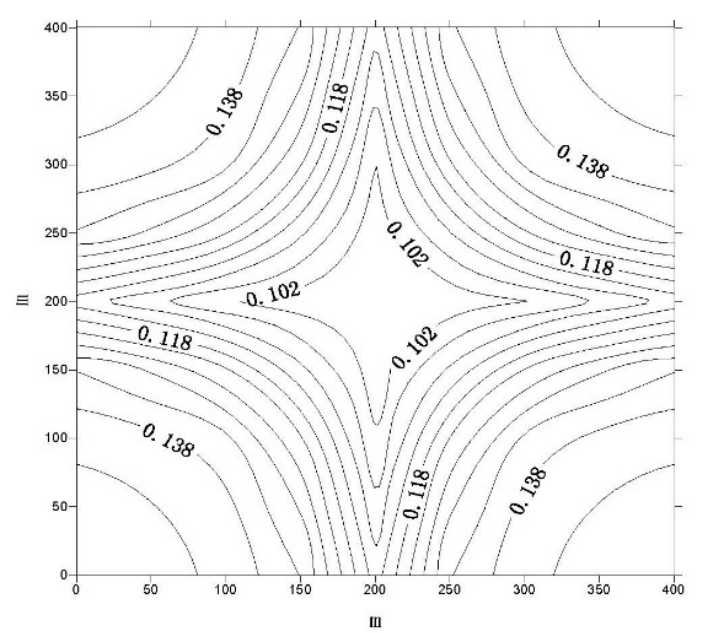

Fig. $4 \mathrm{~b}$ Isogram of the polymer concentration in the second layer, date: $1 / 1 / 2008$

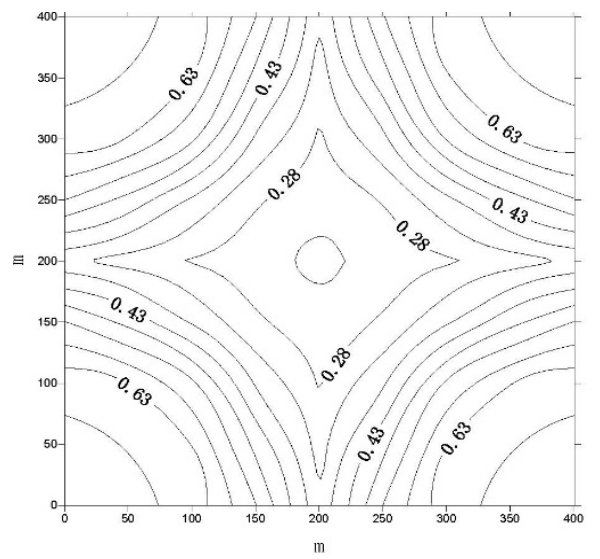

Fig. 5a Isogram of water saturation in the first layer, date: $1 / 1 / 2008$ 


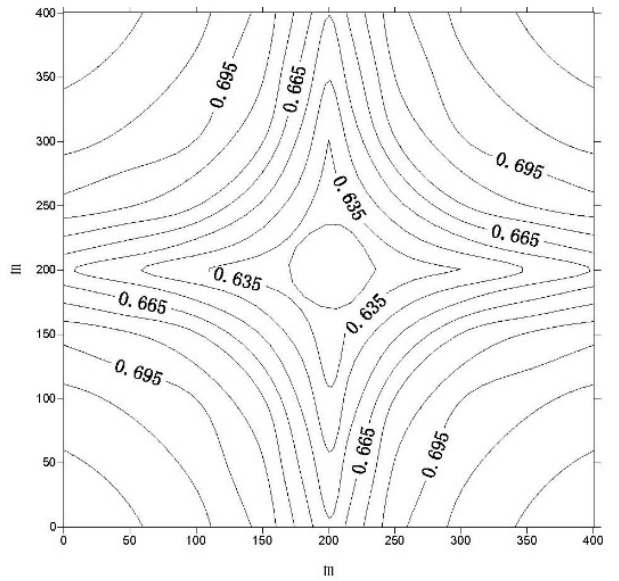

Fig. $5 \mathrm{~b}$ Isogram of water saturation in the second layer, date: 1/1/2008

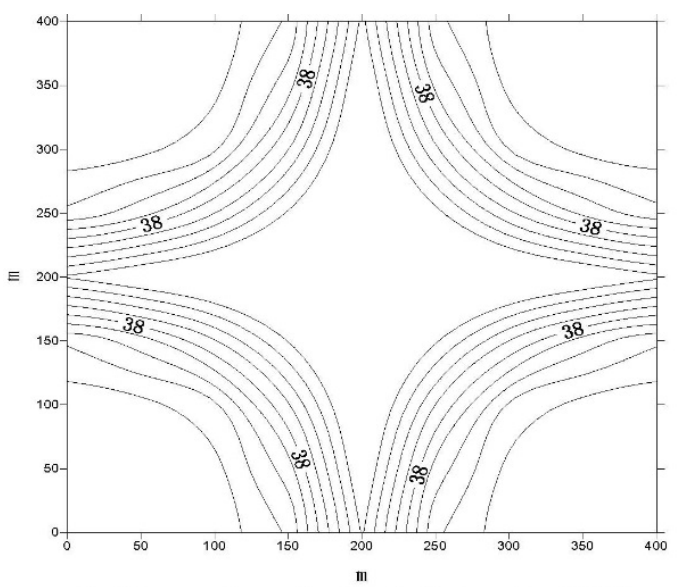

Fig. 6a Isogram of water viscosity in the first layer, date: 1/1/2008

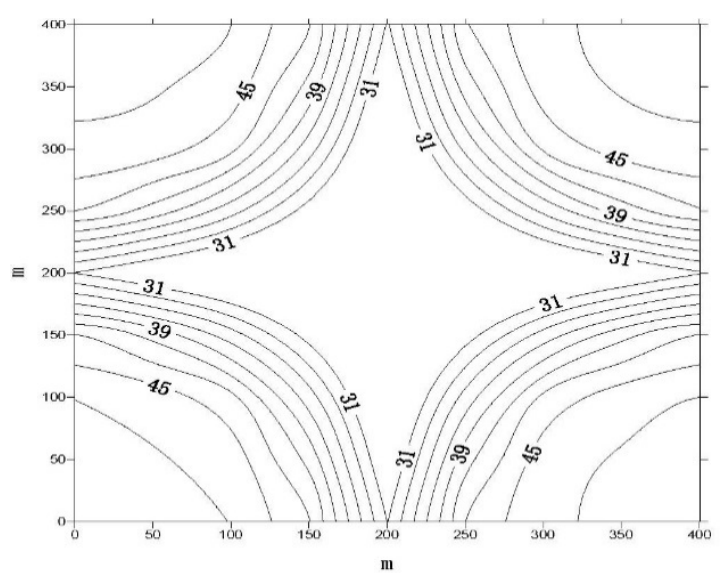

Fig. 6 Isogram of water viscosity in the third layer, date: 1/1/2008

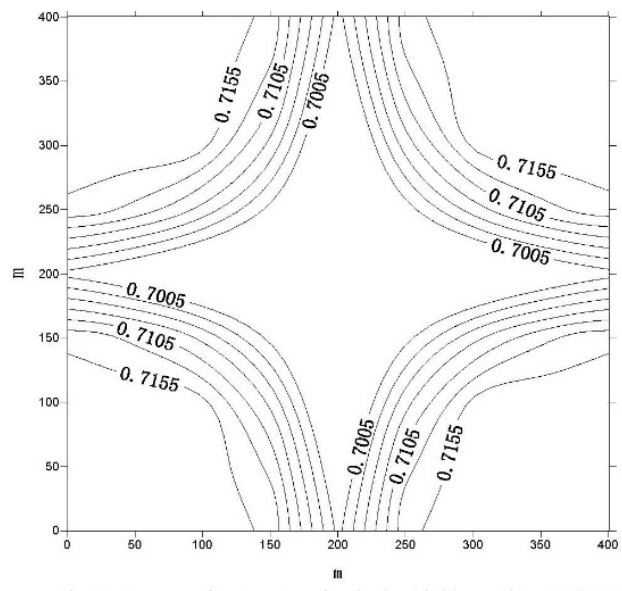

Fig. 5c Isogram of water saturation in the third layer, date: 1/1/2008

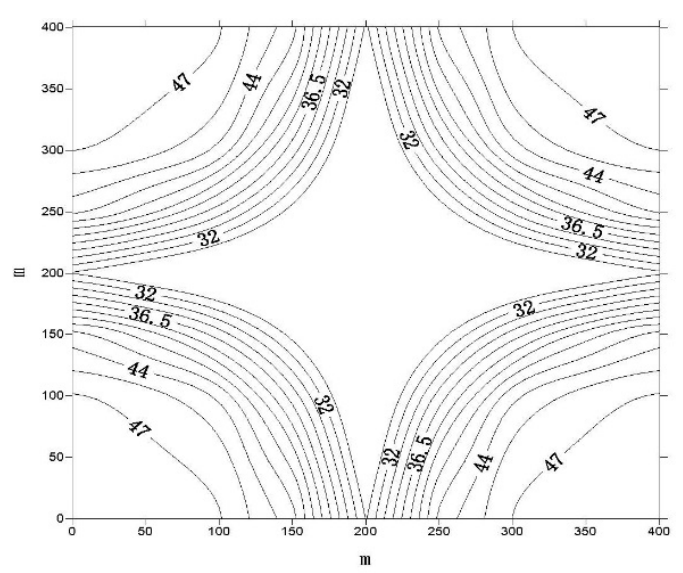

Fig. $6 \mathrm{~b}$ Isogram of water viscosity in the second layer, date: $1 / 1 / 2008$

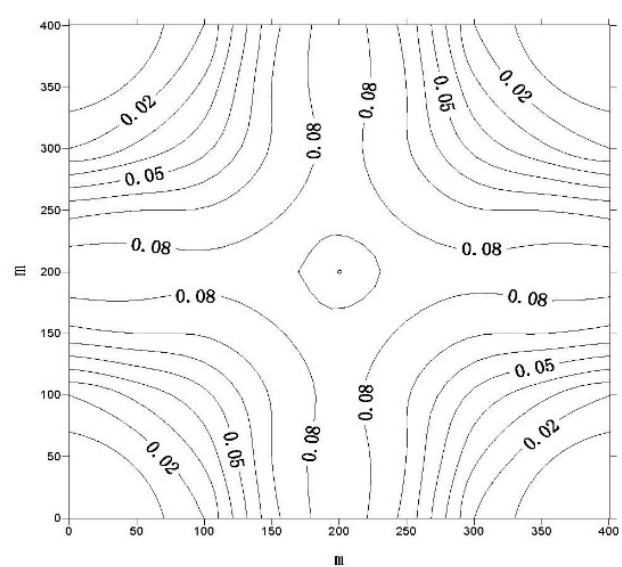

Fig. 7a Isogram of the polymer concentration in the first layer, date: 20/8/2011 


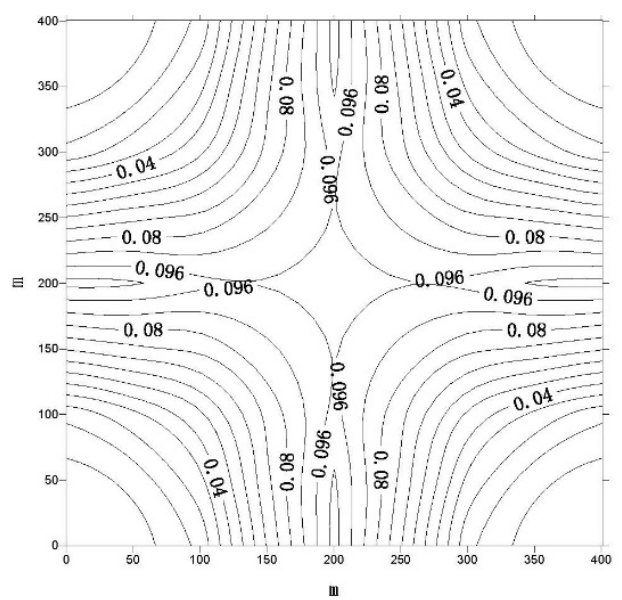

Fig. $7 \mathrm{~b}$ Isogram of the polymer concentration in the second layer, date: 20/8/2011

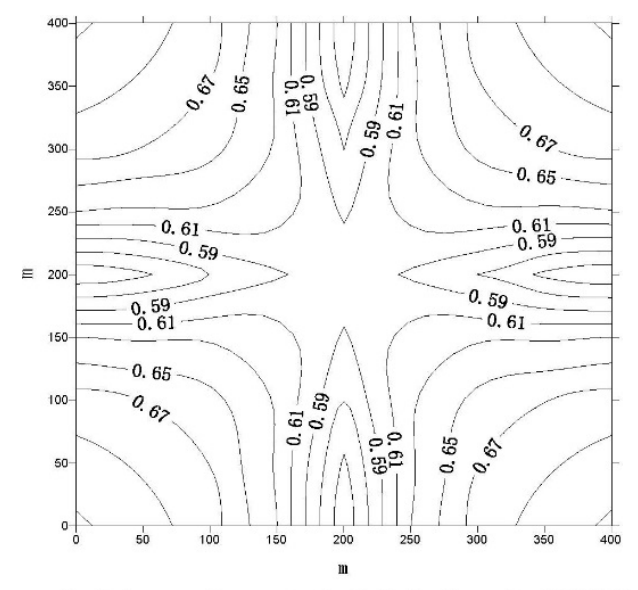

Fig. 8a Isogram of water saturation in the first layer, date: 20/8/2011

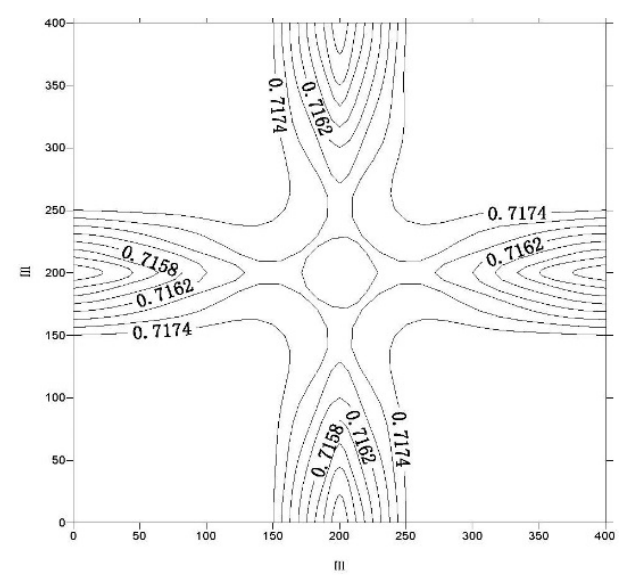

Fig. $8 \mathrm{c}$ Isogram of water saturation in the third layer, date: $20 / 8 / 2011$

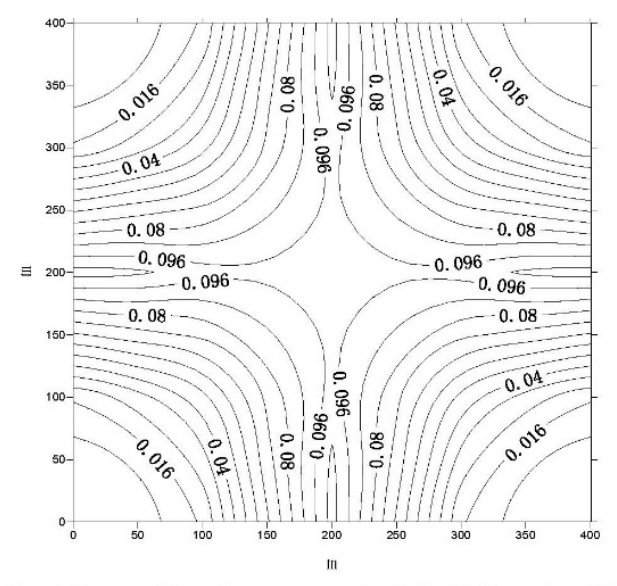

Fig. 7 I Isogram of the polymer concentration in the third layer, date: $20 / 8 / 2011$

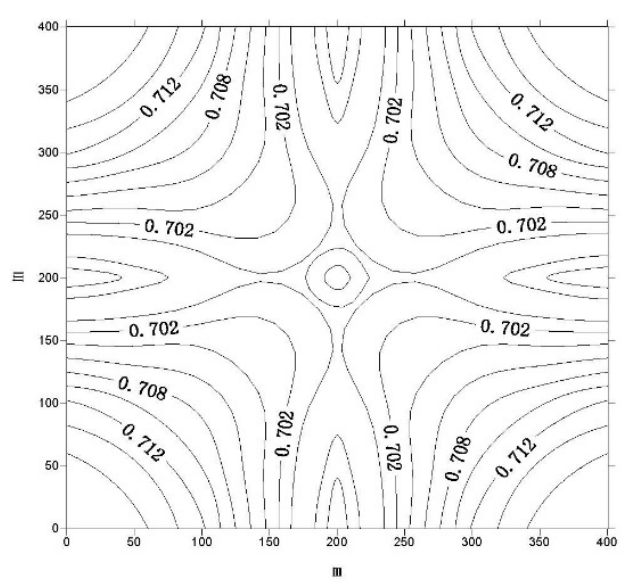

Fig. 8 b Isogram of water saturation in the second layer, date: 20/8/2011

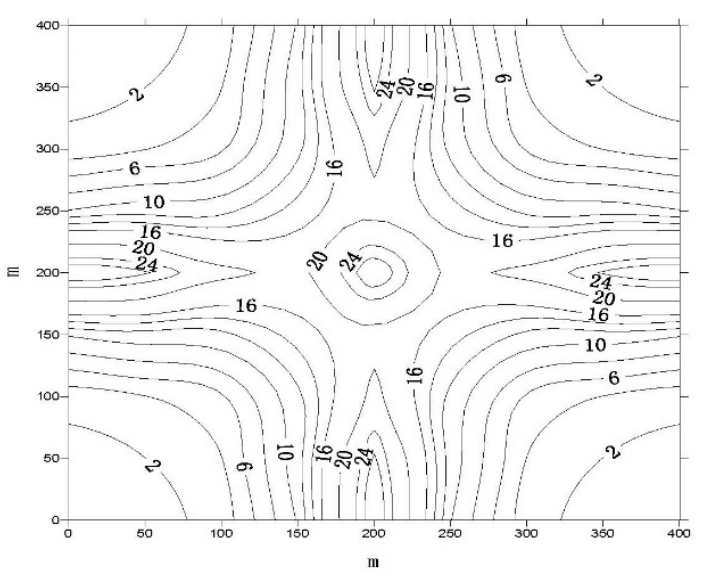

Fig. 9a Isogram of water viscosity in the first layer, date: 20/8/2011 


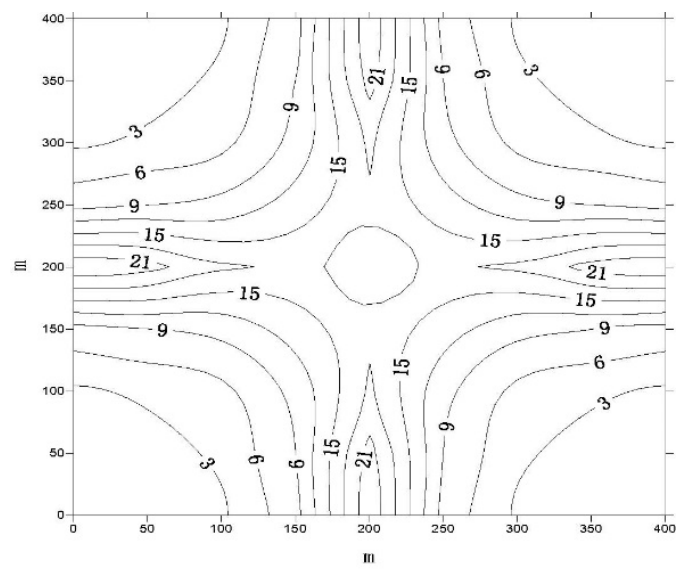

Fig. 9b Isogram of water viscosity in the second layer, date: 20/8/2011

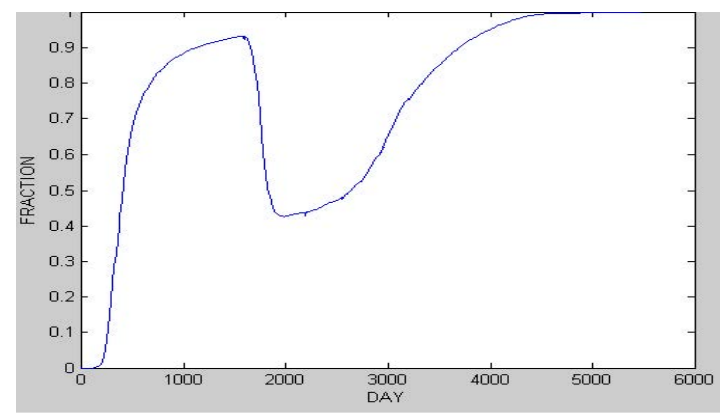

Fig. 10a Sketch of moi sture content curve, the concentration of injected polymer: $2000 \mathrm{mg} / \mathrm{L}$

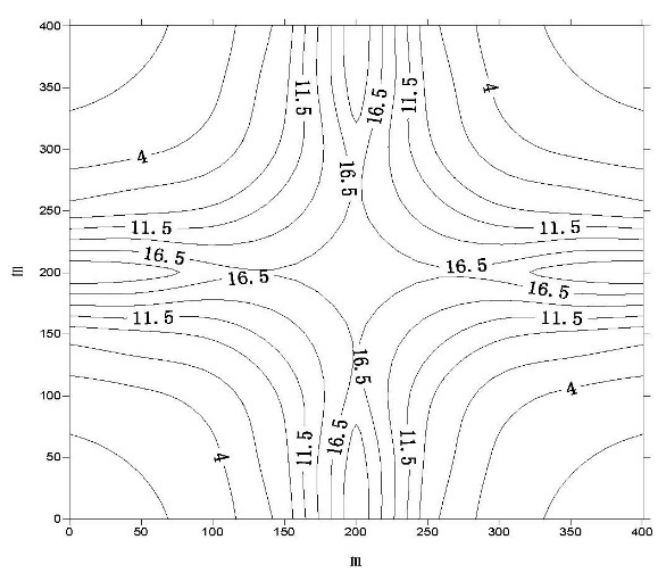

Fig. 9c Isogram of water viscosity in the third layer, date: 20/8/2011

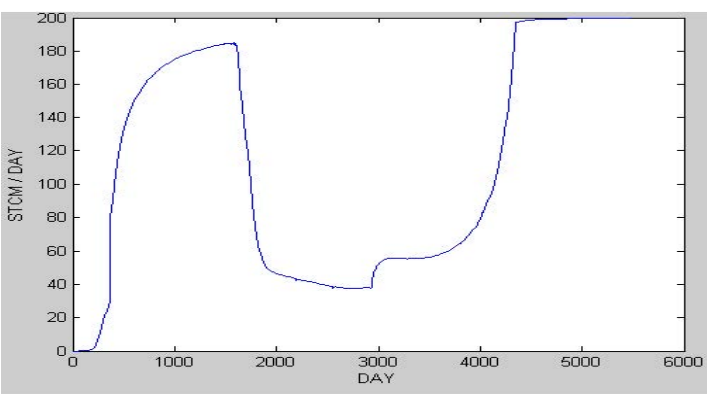

Fig. 10b Sketch of hydraulic discharge curwe, the concentration of injected polymer: $2000 \mathrm{mg} / \mathrm{L}$

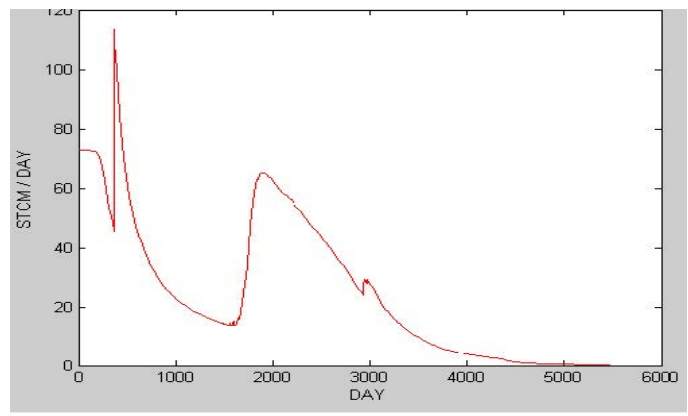

Fig. 10c Sketch of oil pump capacity curve, the concentration of injected polymer: $2000 \mathrm{mg} / \mathrm{L}$

\section{Theoretical Analysis of the Model}

Theoretical analysis of numerical simulation is given for three dimensional three phases (oil, water and gas) polymer flooding in porous media. A simplified model is discussed in theoretical analysis without loss of generality, that is to say a compressible oil water two phase displacement of three dimensional multi-components problem in porous media is discussed in a nonlinear partial differential equations with initial and boundary values (see Ewing, Yuan, \& Li, 1989; Yuan, 2013; Ewing, 1983; Yuan, 2002, 1999, 2003, 2001 ).

$$
\begin{gathered}
d(c) \frac{\partial p}{\partial t}+\nabla \cdot \mathbf{u}=q(x, t), x=\left(x_{1}, x_{2}, x_{3}\right)^{T} \in \Omega, t \in J=(0, T], \\
\mathbf{u}=-a(c) \nabla p, x \in \Omega, t \in J, \\
\Phi(x) \frac{\partial c_{\alpha}}{\partial t}+b_{\alpha}(c) \frac{\partial p}{\partial t}+\mathbf{u} \cdot \nabla c_{\alpha}-\nabla \cdot\left(D \nabla c_{\alpha}\right)=g\left(x, t, c_{\alpha}\right), x \in \Omega, t \in J, \alpha=1,2, \cdots, n_{c}-1,
\end{gathered}
$$

where $p(x, t)$ is the pressure of the mixture, $c_{\alpha}(x, t)$ is the saturation of the $\alpha$-th component, $\alpha=1,2, \cdots, n_{c}$ and 
$n_{c}$ is the number of components. There are $n_{c}-1$ independent components because of $\sum_{\alpha=1}^{n_{c}} c_{\alpha}(x, t)=1$. Let $c(x, t)=\left(c_{1}(x, t), c_{2}(x, t), \ldots, c_{n c-1}(x, t)\right)^{T}$ be the vector function of component saturations, $d(c)=\Phi(x) \sum_{\alpha=1}^{n_{c}} z_{\alpha} c_{\alpha}, \Phi(x)$ be the porosity of rock, $z_{\alpha}$ be the compressible invariant of $\alpha, \boldsymbol{u}$ be Darcy velocity of the mixture, $a(c)=\kappa(x) \mu(c)^{-1}, \kappa(x)$ be the permeability, $\mu(c)$ be the viscosity, $b_{\alpha}(c)=\Phi c_{\alpha}\left\{z_{\alpha}-\sum_{j=1}^{n_{c}} z{ }_{j}{ }_{j}\right\}$, and let $D=D(x)$ be the diffusion parameter. The pressure $p(x, t)$ and the saturation vector $c(x, t)$ are basic unknown functions to compute.

Boundary condition without permeation:

$$
\mathbf{u} \cdot \gamma=0, x \in \partial \Omega,\left(D \nabla c_{\alpha}-c_{\alpha} \mathbf{u}\right) \cdot \gamma=0, x \in \partial \Omega, t \in J, \alpha=1,2, \cdots, n_{c}-1,
$$

where $\gamma$ is the outer normal vector of boundary surface $\partial \Omega$ of $\Omega$.

Initial conditions:

$$
p(x, 0)=p_{0}(x), x \in \Omega, c_{\alpha}(x, 0)=c_{\alpha, 0}(x), x \in \Omega, \alpha=1,2, \cdots, n_{c}-1 .
$$

Douglas (1983) presents a fundamental paper to analyze a type of two dimensional incompressible two-phase displacement problems. Because the computation of modern reservoir exploration and development is of huge scale, its simulation region is large, its simulation time is really long and there are tens of thousands or hundreds of thousands nodes, it is impossible to solve so complicated problems of this type by using common methods. Alternating direction finite difference methods are put forward by Peaceman (1980) and Douglas (1963) and are successfully applied in two dimensional problems but there is substantial difficulty to give theoretical analysis. Using Fourier method Peaceman and Douglas discuss the stability and convergence for the problems with constant coefficients, while this method is not able to generalize in variable coefficient problems. Yanenke (1967), and Marchuk (1990) give many important results on fractional steps methods. Yuan (1999) presents a characteristic finite difference fractional steps method for compressible two-phase displacement problem and discusses convergence analysis. An implicit upwind finite difference fractional steps method is considered for black oil and polymer flooding problem and some substantial improvements are given in this paper. The three dimensional problem is turned into three one-dimensional problems and this can greatly reduce the computation and can solve actual problems. Error estimates in $L^{2}$ norm are presented by using variation, energy analysis, decomposition of high order difference operators and theory and technique of product commutativity.

For simplicity, assume computational domain $\Omega=\{[0,1]\}^{3}$ and the problem is $\Omega$-periodic. The nonpermeation boundary condition can be dropped. Let $h=1 / N, X_{i j k}=(i h, j h, k h)^{T}, t^{n}=n \Delta t$ and $W\left(X_{i j k}, t^{n}\right)=W_{i j k}^{n}$, and let

$$
\begin{gathered}
A_{i+1 / 2, j k}^{n}=\frac{1}{2}\left[a\left(X_{i j k}, C_{i j k}^{n}\right)+a\left(X_{i+1, j k}, C_{i+1, j k}^{n}\right)\right], \\
\delta_{\bar{x}_{1}}\left(A^{n} \delta_{x_{1}} P^{n+1}\right)_{i j k}=h^{-2}\left[A_{i+1 / 2, j k}^{n}\left(\mathrm{P}_{i+1, j k}^{n+1}-\mathrm{P}_{i j k}^{n+1}\right)-A_{i-1 / 2, j k}^{n}\left(\mathrm{P}_{i j k}^{n+1}-\mathrm{P}_{i-1, j k}^{n+1}\right)\right] .
\end{gathered}
$$

Difference operators $A_{i, j+1 / 2, k}^{n}, A_{i j, k+1 / 2}^{n}, \quad \delta_{\bar{x}_{2}}\left(A^{n} \delta_{x_{2}} P^{n+1}\right)_{i j k}$ and $\delta_{\bar{x}_{3}}\left(A^{n} \delta_{x_{3}} P^{n+1}\right)_{i j k}$ can be defined similarly.

The implicit finite difference fractional steps algorithm for the flow equation (20a) is shown as follows,

$$
\begin{gathered}
d\left(C_{i j k}^{n}\right) \frac{P_{i j k}^{n+1 / 3}-P_{i j k}^{n}}{\Delta t}=\delta_{\bar{x}_{1}}\left(A^{n} \delta_{x_{1}} P^{n+1 / 3}\right)_{i j k}+\delta_{\bar{x}_{2}}\left(A^{n} \delta_{x_{2}} P^{n}\right)_{i j k}+\delta_{\bar{x}_{3}}\left(A^{n} \delta_{x_{3}} P^{n}\right)_{i j k}+q\left(X_{i j k}, t^{n+1}\right), 1 \leq i \leq N, \\
d\left(C_{i j k}^{n}\right) \frac{P_{i j k}^{n+2 / 3}-P_{i j k}^{n+1 / 3}}{\Delta t}=\delta_{\bar{x}_{2}}\left(A^{n} \delta_{x_{2}}\left(P^{n+2 / 3}-P^{n}\right)\right)_{i j k}, 1 \leq j \leq N, \\
d\left(C_{i j k}^{n} \frac{P_{i j k}^{n+1}-P_{i j k}^{n+2 / 3}}{\Delta t}=\delta_{\bar{x}_{3}}\left(A^{n} \delta_{x_{3}}\left(P^{n+1}-P^{n}\right)\right)_{i j k}, 1 \leq k \leq N .\right.
\end{gathered}
$$

Darcy velocity $\boldsymbol{U}=\left(U_{1}, U_{2}, U_{3}\right)^{T}$ is computed by the following expression

$$
U_{1, j k k}^{n+1}=-\frac{1}{2}\left[A_{i+1 / 2, j k}^{n} \frac{P_{i+1, j k}^{n+1}-P_{i j k}^{n+1}}{h}+A_{i-1 / 2, j k}^{n} \frac{P_{i j k}^{n+1}-P_{i-1, j k}^{n+1}}{h}\right],
$$

and analogous forms $U_{2, i j k}^{n+1}, U_{3, j k}^{n+1}$ can be derived.

The implicit upwind finite difference fractional steps algorithm for the saturation equations is 


$$
\begin{aligned}
& \Phi_{i j k} \frac{C_{\alpha, j i k}^{n+1 / 3}-C_{\alpha, j i k}^{n}}{\Delta t}=\delta_{\bar{x}_{1}}\left(D \delta_{x_{1}} C_{\alpha}^{n+1 / 3}\right)_{i j k}+\delta_{\bar{x}_{2}}\left(D \delta_{x_{2}} C_{\alpha}^{n}\right)_{i j k}+\delta_{\bar{x}_{3}}\left(D \delta_{x_{3}} C_{\alpha}^{n}\right)_{i j k} \\
& -b_{\alpha}\left(C_{\alpha, i j k}^{n}\right) \frac{P_{i j k}^{n+1}-P_{i j k}^{n}}{\Delta t}+g\left(X_{i j k}, t^{n}, C_{\alpha, j i k}^{n}\right), 1 \leq i \leq N, \alpha=1,2, \cdots, N_{c}-1, \\
& \Phi_{i j k} \frac{C_{\alpha, j i k}^{n+2 / 3}-C_{\alpha, j i k}^{n+1 / 3}}{\Delta t}=\delta_{\bar{x}_{2}}\left(D \delta_{x_{2}}\left(C_{\alpha}^{n+2 / 3}-C_{\alpha}^{n}\right)\right)_{i j k}, 1 \leq j \leq N, \alpha=1,2, \cdots, N_{c}-1, \\
& \Phi_{i j k} \frac{C_{\alpha, j i k}^{n+1}-C_{\alpha, j i k}^{n+2 / 3}}{\Delta t}=\delta_{\bar{x}_{3}}\left(D \delta_{x_{3}}\left(C_{\alpha}^{n+1}-C_{\alpha}^{n}\right)\right)_{i j k}-\sum_{\beta=1}^{3} \delta_{U_{\beta}^{n+1}, x_{\beta}} C_{\alpha, j i k}^{n+1}, 1 \leq k \leq N, \alpha=1,2, \cdots, N_{c}-1,
\end{aligned}
$$

where

Initial approximation:

$$
\delta_{U_{\beta}^{n+1}, x_{\beta}} C_{\alpha, j i k}^{n+1}=U_{\beta, j i k}^{n+1}\left\{H\left(U_{\beta, j i k}^{n+1}\right) \delta_{\bar{x}_{\beta}}+\left(1-H\left(U_{\beta, j i k}^{n+1}\right)\right) \delta_{x_{\beta}}\right\} C_{\alpha, j i k}^{n+1}, \beta=1,2,3, \alpha=1,2, \cdots, n_{c}-1, \quad H(z)=\left\{\begin{array}{l}
1, z \geq 0, \\
0, z<0 .
\end{array}\right.
$$

$$
P_{i j k}^{0}=p_{0}\left(X_{i j k}\right), C_{\alpha, j i k}^{0}=c_{\alpha, 0}\left(X_{i j k}\right), 1 \leq i, j, k \leq N, \alpha=1,2, \cdots, n_{c}-1 .
$$

The implicit upwind fractional steps algorithm runs as follows. Given $\left\{P_{i j k}^{n}, C_{\alpha, j k k}^{n}, \alpha=1,2, \cdots, n_{c}-1\right\}$, the values of transition layer by (24a) and speedup method in $x_{1}$ direction firstly. Then $\left\{P_{i j k}^{n+2 / 3}\right\}$ and $\left\{P_{i j k}^{n+1}\right\}$ are computed by (24b) and by (24c), respectively. Applying (25) we can have the numerical values of velocity $\left\{U_{i j k}^{n+1}\right\}$. Secondly, $\left\{C_{\alpha, i j k}^{n+1 / 3}\right\}$ is computed by (26a) and speedup method in $x_{1}$ direction. $\left\{C_{\alpha, j i k}^{n+2 / 3}\right\}$ and $\left\{C_{\alpha, j k}^{n+1}\right\}$ are obtained similarly by (26b) and (26c). Notice the problem is positive definite, so the solution of (24)-(26) exists and is unique.

Let $\pi=p-P$ and $\xi_{\alpha}=c_{\alpha}-C_{\alpha}$ where $p, c_{\alpha}$ are exact solutions and $P, C_{\alpha}$ are numerical solutions. The pressure equation is considered firstly, and its equivalent difference expression is

$$
\begin{aligned}
& d\left(C_{i j k}^{n}\right) \frac{P_{i j k}^{n+1}-P_{i j k}^{n}}{\Delta t}-\nabla_{h}\left(A^{n} \nabla_{h} P^{n+1}\right)_{i j k}=q\left(X_{i j k}, t^{n+1}\right)-(\Delta t)^{2}\left\{\delta_{\bar{x}_{1}}\left(A^{n} \delta_{x_{1}}\left(d^{-1}\left(C^{n}\right) \delta_{\bar{x}_{2}}\left(A^{n} \delta_{x_{2}}\right)\right)\right)\right. \\
& +\delta_{\overline{x_{1}}}\left(A^{n} \delta_{x_{1}}\left(d^{-1}\left(C^{n}\right) \delta_{\bar{x}_{3}}\left(A^{n} \delta_{\overline{x_{3}}}\right)\right)\right)+\delta_{\overline{\bar{x}_{2}}}\left(A^{n} \delta_{x_{2}}\left(d^{-1}\left(C^{n}\right) \delta_{\bar{x}_{3}}\left(A^{n} \delta_{x_{3}}\right)\right)\right\} d_{t} P_{i j k}^{n} \\
& +(\Delta t)^{3} \delta_{\bar{x}_{1}}\left(A ^ { n } \delta _ { \overline { x } _ { 1 } } \left(d ^ { - 1 } ( C ^ { n } ) \delta _ { \overline { x } _ { 2 } } \left(A^{n} \delta_{x_{2}}\left(d^{-1}\left(C^{n}\right) \delta_{\bar{x}_{3}}\left(A^{n} \delta_{\bar{x}_{3}} d_{t} P^{n}\right) \cdots\right)_{i j k}, 1 \leq i, j, k \leq N .\right.\right.\right.
\end{aligned}
$$

Then the error equation of the pressure is

$$
\begin{aligned}
& d\left(C_{i j k}^{n}\right) \frac{\pi_{i j k}^{n+1}-\pi_{i j k}^{n}}{\Delta t}-\nabla_{h}\left(A^{n} \nabla_{h} \pi^{n+1}\right)_{i j k}=-(\Delta t)^{2}\left\{\delta_{\bar{x}_{1}}\left(A^{n} \delta_{x_{1}}\left(d^{-1}\left(C^{n}\right) \delta_{\bar{x}_{2}}\left(A^{n} \delta_{x_{2}}\right)\right)\right)+\delta_{\bar{x}_{1}}\left(A^{n} \delta_{x_{1}}\left(d^{-1}\left(C^{n}\right) \delta_{\bar{x}_{3}}\left(A^{n} \delta_{\bar{x}_{3}}\right)\right)\right)\right. \\
& +\delta_{\overline{x_{2}}}\left(A^{n} \delta_{x_{2}}\left(d^{-1}\left(C^{n}\right) \delta_{\overline{\bar{y}_{3}}}\left(A^{n} \delta_{x_{3}}\right)\right)\right\} d_{t} \pi_{i j k}^{n}+(\Delta t)^{3} \delta_{\bar{x}_{1}}\left(A ^ { n } \delta _ { x _ { 1 } } \left(d ^ { - 1 } ( C ^ { n } ) \delta _ { \overline { \overline { z } _ { 2 } } } \left(A^{n} \delta_{x_{2}}\left(d^{-1}\left(C^{n}\right) \delta_{\bar{x}_{3}}\left(A^{n} \delta_{\bar{x}_{3}} d_{t} P^{n}\right) \cdots\right)_{i j k}\right.\right.\right. \\
& +(\Delta t)^{2}\left\{\delta_{\bar{x}_{1}}\left(A^{n} \delta_{x_{1}}\left(d^{-1}\left(C^{n}\right) \delta_{\bar{x}_{2}}\left(A^{n} \delta_{x_{2}}\right)\right)\right)+\cdots\right\} d_{t} p_{i j k}^{n} \\
& -(\Delta t)^{3} \delta_{\bar{x}_{1}}\left(A ^ { n } \delta _ { x _ { 1 } } \left(d ^ { - 1 } ( C ^ { n } ) \delta _ { \overline { x } _ { 2 } } \left(A^{n} \delta_{x_{2}}\left(d^{-1}\left(C^{n}\right) \delta_{\bar{x}_{3}}\left(A^{n} \delta_{\bar{x}_{3}} d_{t} p^{n}\right) \cdots\right)_{i j k}+\sigma_{i j k}^{n+1}, 1 \leq i, j, k \leq N,\right.\right.\right.
\end{aligned}
$$

where $d_{t} \pi^{n}=\left(\pi^{n+1}-\pi^{n}\right) / \Delta t,\left|\sigma_{i j k}^{n+1}\right| \leq M\left\{h^{2}+\Delta t\right\}$.

Multiplying both sides of error equation (29) by $\delta_{t} \pi^{n}=\pi^{n+1}-\pi^{n}=d_{t} \pi^{n} \Delta t$, summation by parts, and obtaining an inner product form

$$
\begin{aligned}
& \left\langle d\left(C^{n}\right) d_{t} \pi^{n}, d_{t} \pi^{n}\right\rangle \Delta t+\frac{1}{2}\left\{\left\langle A^{n} \nabla_{h} \pi^{n+1}, \nabla_{h} \pi^{n+1}\right\rangle-\left\langle A^{n} \nabla_{h} \pi^{n}, \nabla_{h} \pi^{n}\right\rangle\right\} \\
& \leq M\left\{h^{4}+(\Delta t)^{2}\right\} \Delta t+\varepsilon\left|d_{t} \pi^{n}\right|_{0}^{2} \Delta t-(\Delta t)^{2}\left\{\left\langle\delta_{\overline{x_{1}}}\left(A^{n} \delta_{\bar{x}_{1}}\left(d^{-1}\left(C^{n}\right) \delta_{\bar{x}_{2}}\left(A^{n} \delta_{x_{2}} d_{t} \pi^{n}\right)\right)\right), d_{t} \pi^{n}\right\rangle\right. \\
& \left.+\left\langle\delta_{\bar{x}_{1}}\left(A^{n} \delta_{x_{1}}\left(d^{-1}\left(C^{n}\right) \delta_{\bar{x}_{3}}\left(A^{n} \delta_{\bar{x}_{3}} d_{t} \pi^{n}\right)\right)\right), d_{t} \pi^{n}\right\rangle+\left\langle\delta_{\bar{x}_{2}}\left(A^{n} \delta_{x_{2}}\left(d^{-1}\left(C^{n}\right) \delta_{\overline{x_{3}}}\left(A^{n} \delta_{x_{3}} d_{t} \pi^{n}\right)\right)\right), d_{t} \pi^{n}\right\rangle\right\} \Delta t \\
& +(\Delta t)^{3}\left\langle\delta _ { \overline { x } _ { 1 } } \left(A ^ { n } \delta _ { \overline { x } _ { 1 } } \left( d^{-1}\left(C^{n}\right) \delta_{\bar{x}_{2}}\left(A^{n} \delta_{x_{2}}\left(d^{-1}\left(C^{n}\right) \delta_{\bar{x}_{3}}\left(A^{n} \delta_{\bar{x}_{3}} d_{t} \pi^{n}\right) \cdots\right), d_{t} \pi^{n}\right\rangle \Delta t .\right.\right.\right.
\end{aligned}
$$

Then it follows from (30) by complicated estimates,

$$
\left|d_{t} \pi^{n}\right|_{0}^{2} \Delta t+\frac{1}{2}\left\{\left\langle A^{n} \nabla_{h} \pi^{n+1}, \nabla_{h} \pi^{n+1}\right\rangle-\left\langle A^{n} \nabla_{h} \pi^{n}, \nabla_{h} \pi^{n}\right\rangle\right\} \leq M\left\{\left|\pi^{n+1}\right|_{1}^{2}+\left|\pi^{n}\right|_{1}^{2}+h^{4}+(\Delta t)^{2}\right\} \Delta t .
$$

Secondly an error analysis proceeds for the saturation equations. Eq. (26) is rewritten in the following equivalent 
form

$$
\begin{aligned}
& \Phi_{i j k} \frac{C_{\alpha, j i k}^{n+1}-C_{\alpha, j k k}^{n}}{\Delta t}-\nabla_{h}\left(D \nabla_{h} C_{\alpha}^{n+1}\right)_{i j k}^{n+1}=-\sum_{\beta=1}^{3} \delta_{U_{\beta}^{n+1}, x_{\beta}} C_{\alpha, j i k}^{n+1}+b_{\alpha}\left(C_{i j k}\right) \frac{P_{i j k}^{n+1}-P_{i j k}^{n}}{\Delta t}+g\left(X_{i j k}, t^{n+1}, C_{\alpha, i j k}^{n+1}\right) \\
& -(\Delta t)^{2}\left\{\delta_{\bar{x}_{1}}\left(D \delta_{x_{1}}\left(\Phi^{-1} \delta_{\bar{x}_{2}}\left(D \delta_{x_{2}}\right)\right)\right)+\delta_{\bar{x}_{1}}\left(D \delta_{x_{1}}\left(\Phi^{-1} \delta_{\bar{x}_{3}}\left(D \delta_{x_{3}}\right)\right)\right)+\delta_{\bar{x}_{2}}\left(D \delta_{x_{2}}\left(\Phi^{-1} \delta_{\bar{x}_{3}}\left(D \delta_{x_{3}}\right)\right)\right)\right\} d_{t} C_{\alpha, j i k}^{n} \\
& +(\Delta t)^{3} \delta_{\bar{x}_{1}}\left(D \delta _ { x _ { x _ { 1 } } } \left(\Phi ^ { - 1 } \delta _ { \overline { x } _ { 2 } } \left(D \delta_{x_{2}}\left(\Phi^{-1} \delta_{\bar{x}_{3}}\left(D \delta_{x_{3}} d_{t} C_{\alpha}^{n}\right) \cdots\right)_{i j k}, 1 \leq i, j, k \leq N, \alpha=1,2, \cdots, n_{c}-1 .\right.\right.\right.
\end{aligned}
$$

From (20c) and (32),

$$
\begin{aligned}
& \Phi_{i j k} \frac{\xi_{\alpha, j i k}^{n+1}-\xi_{\alpha, j i k}^{n}}{\Delta t}-\nabla_{h}\left(D \nabla_{h} \xi_{\alpha}\right)_{j i k}^{n+1}=-\sum_{\beta=1}^{3}\left[\delta_{U_{\beta}^{n+1}, x_{\beta}} C_{\alpha, j i k}^{n+1}-\delta_{u_{\beta}^{n+1}, x_{\beta}} c_{\alpha, j i k}^{n+1}\right]+g\left(X_{i j k}, t^{n+1}, C_{\alpha, i j k}^{n+1}\right)-g\left(X_{i j k}, t^{n}, C_{\alpha, j i k}^{n}\right) \\
& +b_{\alpha}\left(C_{i j k}^{n}\right) \frac{\pi_{i j k}^{n+1}-\pi_{i j k}^{n}}{\Delta t}-\left[b_{\alpha}\left(C_{i j k}^{n+1}\right)-b_{\alpha}\left(c_{i j k}^{n}\right)\right] \frac{p_{i j k}^{n+1}-p_{i j k}^{n}}{\Delta t}+(\Delta t)^{2}\left\{\delta_{\bar{x}_{1}}\left(D \delta_{x_{1}}\left(\Phi^{-1} \delta_{\bar{x}_{2}}\left(D \delta_{x_{2}}\right)\right)\right)\right. \\
& +\delta_{\bar{x}_{1}}\left(D \delta_{x_{1}}\left(\Phi^{-1} \delta_{\bar{x}_{3}}\left(D \delta_{x_{3}}\right)\right)\right)+\delta_{\bar{x}_{2}}\left(D \delta_{x_{2}}\left(\Phi^{-1} \delta_{\bar{x}_{3}}\left(D \delta_{x_{3}}\right)\right)\right) d_{t} \xi_{\alpha, j i k}^{n} \\
& +(\Delta t)^{3} \delta_{\bar{x}_{1}}\left(D \delta _ { x _ { 1 } } \left(\Phi ^ { - 1 } \delta _ { \overline { z } _ { 2 } } \left(D \delta_{x_{2}}\left(\Phi^{-1} \delta_{\bar{x}_{3}}\left(D \delta_{x_{3}} d_{t} \xi_{\alpha}^{n}\right) \cdots\right)_{i j k}+\varepsilon_{\alpha, j i k}^{n+1}, 1 \leq i, j, k \leq N, \alpha=1,2, \cdots, n_{c}-1,\right.\right.\right.
\end{aligned}
$$

where $\left|\varepsilon_{\alpha, i j k}^{n+1}\right| \leq M\{h+\Delta t\}$.

Testing the above equation by $\delta_{t} \xi_{\alpha}^{n}=\xi_{\alpha}^{n+1}-\xi_{\alpha}^{n}=d_{t} \xi_{\alpha}^{n} \Delta t$, summation by parts, and obtaining an inner product form

$$
\begin{aligned}
& \left\langle\Phi d_{t} \xi_{\alpha}^{n}, d_{t} \xi_{\alpha}^{n}\right\rangle+\frac{1}{2}\left\{\left\langle D \nabla_{h} \xi_{\alpha}^{n+1}, \nabla_{h} \xi_{\alpha}^{n+1}\right\rangle-\left\langle D \nabla_{h} \xi_{\alpha}^{n}, \nabla_{h} \xi_{\alpha}^{n}\right\rangle\right\} \\
& \leq M\left\{\left|\xi^{n}\right|_{0}^{2}+\left|\xi^{n+1}\right|_{0}^{2}+\left|\pi^{n+1}\right|_{1}^{2}+h^{2}+(\Delta t)^{2}\right\} \Delta t+\varepsilon\left|d_{t} \xi_{\alpha}^{n}\right|_{0}^{2} \Delta t+\left\langle b_{\alpha}\left(C^{n}\right) d_{t} \pi^{n}, d_{t} \pi^{n}\right\rangle \\
& -(\Delta t)^{2}\left\{\left\langle\delta_{\bar{x}_{1}}\left(D \delta_{x_{1}}\left(\Phi^{-1} \delta_{\bar{x}_{2}}\left(D \delta_{x_{2}} d_{t} \xi_{\alpha}^{n}\right)\right)\right), d_{t} \xi_{\alpha}^{n}\right\rangle\right. \\
& \left.+\left\langle\delta_{\bar{x}_{1}}\left(D \delta_{x_{1}}\left(\Phi^{-1} \delta_{\bar{x}_{3}}\left(D \delta_{\bar{x}_{3}} d_{t} \xi_{\alpha}^{n}\right)\right)\right), d_{t} \xi_{\alpha}^{n}\right\rangle+\left\langle\delta_{\bar{x}_{2}}\left(D \delta_{x_{2}}\left(\Phi^{-1} \delta_{\bar{x}_{3}}\left(D \delta_{x_{3}} d_{t} \xi_{\alpha}^{n}\right)\right)\right), d_{t} \xi_{\alpha}^{n}\right\rangle\right\} \Delta t \\
& +(\Delta t)^{3}\left\langle\delta _ { \overline { x } _ { 1 } } \left(D \delta _ { x _ { 1 } } \left(\Phi^{-1} \delta_{\bar{x}_{2}}\left(D \delta_{x_{2}}\left(\Phi^{-1} \delta_{\bar{x}_{3}}\left(D \delta_{\bar{x}_{3}} d_{t} \xi_{\alpha}^{n}\right) \cdots\right), d_{t} \xi_{\alpha}^{n}\right\rangle \Delta t, \alpha=1,2, \cdots, n_{c}-1,\right.\right.\right.
\end{aligned}
$$

where . $\quad$. It follows form (34)

$$
\left|\xi^{n}\right|_{0}^{2}=\sum_{\alpha=1}^{n_{c}-1}\left|\xi_{\alpha}^{n}\right|_{0}^{2}\left|d_{t} \xi_{\alpha}^{n}\right|_{0}^{2} \Delta t+\left\langle D \nabla_{h} \xi_{\alpha}^{n+1}, \nabla_{h} \xi_{\alpha}^{n+1}\right\rangle-\left\langle D \nabla_{h} \xi_{\alpha}^{n}, \nabla_{h} \xi_{\alpha}^{n}\right\rangle \leq M\left\{\left|\xi^{n}\right|_{1}^{2}+\left|\xi^{n+1}\right|_{1}^{2}+\left|\pi^{n+1}\right|_{1}^{2}+h^{2}+(\Delta t)^{2}\right\} \Delta t
$$

Note $\pi^{0}=0, \xi_{\alpha}^{0}=0\left(\alpha=1,2, \cdots, n_{c}-1\right)$, and

$$
\left|\pi^{L+1}\right|_{0}^{2} \leq \varepsilon \sum_{n=0}^{L}\left|d_{t} \pi^{n}\right|_{0}^{2} \Delta t+M \sum_{n=0}^{L}\left|\pi^{n}\right|_{0}^{2} \Delta t,\left|\xi_{\alpha}^{L+1}\right|_{0}^{2} \leq \varepsilon \sum_{n=0}^{L}\left|d_{t} \xi_{\alpha}^{n}\right|_{0}^{2} \Delta t+M \sum_{n=0}^{L}\left|\xi_{\alpha}^{n}\right|_{0}^{2} \Delta t .
$$

Summing on $0 \leq n \leq L$ for (31) and (35) with respect to $t$, and summing $1 \leq \alpha \leq n_{c}-1$ for (35) with respect to $\alpha$,

$$
\begin{array}{r}
\sum_{n=0}^{L}\left|d_{t} \pi^{n}\right|_{0}^{2} \Delta t+\left|\pi^{L+1}\right| \leq \varepsilon \sum_{n=0}^{L}\left|d_{t} \xi^{n}\right|_{0}^{2} \Delta t+M\left\{h^{2}+(\Delta t)^{2}+\sum_{n=1}^{L}\left|\pi^{n+1}\right|_{1}^{2} \Delta t\right\}, \\
\sum_{n=0}^{L}\left|d_{t} \xi^{n}\right|_{0}^{2} \Delta t+\left|\xi^{L+1}\right| \leq M\left\{h^{2}+(\Delta t)^{2}+\sum_{n=1}^{L}\left[\left|\xi^{n+1}\right|_{1}^{2}+\left|\pi^{n}\right|_{1}^{2}\right] \Delta t\right\},
\end{array}
$$

where $\left|d_{t} \xi^{n}\right|_{0}^{2}=\sum_{\alpha=1}^{n_{c}-1}\left|d_{t} \xi_{\alpha}^{n}\right|_{0}^{2}, \cdots$.

Collecting (36a) and (36b),

$$
\sum_{n=0}^{L}\left\{\left|d_{t} \xi^{n}\right|_{0}^{2}+\left|d_{t} \pi^{n}\right|_{0}^{2}\right\} \Delta t+\left|\xi^{L+1}\right|_{1}^{2}+\left|\pi^{L+1}\right|_{1}^{2} \leq M\left\{h^{2}+(\Delta t)^{2}\right\} .
$$

Theorem Suppose that the exact solutions of (20)-(22) are suitably smooth. Applying implicit upwind difference fractional steps method (24)-(26) to compute layer by layer, we can conclude the following error estimates,

$$
\|p-P\|_{I^{\circ}\left(J ; h^{1}\right)}+\sum_{\alpha=1}^{n_{c}-1}\left\|c_{\alpha}-C_{\alpha}\right\|_{\bar{L}^{\infty}\left(J ; h^{\prime}\right)}+\left\|d_{t}(p-P)\right\|_{\bar{L}^{2}\left(J ; i^{2}\right)}+\sum_{\alpha=1}^{n_{c}-1}\left\|d_{t}\left(c_{\alpha}-C_{\alpha}\right)\right\|_{\bar{L}^{2}\left(J ; i^{2}\right)} \leq M^{*}\{h+\Delta t\}
$$

\section{Discussion}

Theory, method and application of numerical simulation of three-dimensional three-phase (water, oil and gas) 
percolation mechanics of polymer flooding in porous media are discussed in this paper consisting of six sections. Summary is first stated about our project. Mathematical model of permeation fluid mechanics is presented in the first section. A full implicit numerical scheme and implicit/explicit algorithm for the pressure and the saturation are given, and an upwind difference fractional steps algorithm based on upstream sequence is structured in the second section. A type of software applicable in major industries has been accomplished, mostly carried out with the spacial step of ten-meters, tens of thousands nodes and tens of years simulation period in the third section. Some experimental tests occurring successfully in major oil fields such as Daqing Oilfield, Shengli Oilfield and Dagang Oilfield, are illustrated in the fourth section. Numerical analysis proceeds for the model problem and precise theoretical results are stated on mathematical and mechanical consideration in the fifth section.

\section{Acknowledgments}

The authors express their deep appreciation to prof. J. Douglas Jr, prof. R. E. Ewing and prof. Jiang Lishang for their many helpful suggestions in the serial of research of chemical production.

\section{References}

Douglas, Jr. J. (1983). Finite difference methods for two-phase incompressible flow in porous media. SIAM. J. Numer. Anal., 20(4), 681-696. http://dx.doi.org/10.1137/0720046

Douglas, Jr. J., \& Gun, J. E. (1963). Two order correct difference analogues for the equation of multidimensional heat flow. Math. Comp., 81, 71-80. http://dx.doi.org/10.1090/S0025-5718-1963-0149676-2

Ewing, R. E. (1983). The Mathematics of Reservoir Simulation. SIAM, Philadelphia. http://dx.doi.org/10.1137/1.9781611971071

Ewing, R. E., Yuan, Y. R., \& Li, G. (1989). Finite element for chemical-flooding simulation. Proceeding of the 7th International conference finite element method in flow problems, 1264-1271. The University of Alabama in Huntsville, Huntsville, Alabama: UAHDRESS.

Marchuk, G. I. (1990). Splitting and Alternating Direction Methods. In Ciarlet, P. G., \& Lions, J. L., (Eds.), Handbook of Numerical Analysis (pp. 197-400). Paris: Elservier Science Publishers BV.

Peaceman, D. W. (1980). Fundamental of Numerical Reservoir Simulation. Amsterdam: Elsevier.

Yanenke, M. M. (1967). The Method for Fractional Steps. Berlin, Springer-Verlag.

Yuan, Y. R. (1993). The characteristic finite difference method for enhanced oil recovery simulation and $\mathrm{L}^{2}$ estimates. Science in China (Series A), 11, 1296-1307.

Yuan, Y. R. (1993). The characteristic-mixed finite element method for enhanced oil recovery simulation and optimal order L ${ }^{2}$ error estimate. Chinese Science Bulletin, 21, 1761-1766.

Yuan, Y. R. (1994). The characteristic mixed finite element method and analysis for two dimensional chemical-flooding reservoir simulation. Acta Mathematicae Applicatae Sinica, 1, 118-131.

Yuan, Y. R. (1999). The characteristic finite difference fractional steps method for compressible two-phase displacement problem. Science in China (Series A), 1, 48-57. http://dx.doi.org/10.1137/1.9781611971071

Yuan, Y. R. (2000). Finite element method and analysis for chemical-flow simulation. Systems Science and Mathematical Sciences, 3, 302-308

Yuan, Y. R. (2001). Characteristic finite difference fractional steps methods for three-dimensional compressible multi-component displacement problem. Acta Mathematicae Applicatae Sinica, 2, 242-249.

Yuan, Y. R. (2002). The upwind difference method for compressible two-phase displacement problem. Acta Mathematicae Applicatae Sinica, 3, 484-496.

Yuan, Y. R. (2003). The upwind finite difference fractional steps methods for two-phase compressible flow in porous media. Numer Methods Partial Differential Eq., 19, 67-88. http://dx.doi.org/10.1002/num.10036

Yuan, Y. R. (2013). Theory and application of numerical simulation of energy sources, basis of numerical simulation of chemical production (tertiary oil recovery). Chapter, 3, 257-304. Beijing: Science Press.

Yuan, Y. R., Yang, D. P., \& Qi, L. Q. (1998). Research on algorithms of applied software of the polymer. Qinlin Gang (editor in chief), Proceedings on chemical flooding, Beijing: Petroleum Industry Press, 246-253.

\section{Notes}

Note 1. Institute of Mathematics, Shandong University, Exploration Institute of Daqing Petroleum 
Administration. Research and application of the polymer flooding software (summary of "Eighth-Five" national key science and technology program, Grant No. 85-203-01-08), 1995.10.

Note 2. China National Petroleum Corporation. Evaluation report of executive condition of "Eighth-Five" national key science and technology program (Grant no. 85-203-01-08).1995.10.

Note 3. Institute of Mathematics, Shandong University, Exploration and development of Daqing Petroleum Corporation. Modification of solving mathematical models of the polymer and improvement of reservoir description. 2006.

Note 4. Institute of Mathematics, Shandong University, Shengli Oilfield Branch, China Petroleum \& Chemical Corporation. Research on key technology of high temperature and high salinity chemical agent displacement, Chapter 4,§4.1 Numerical method, 83-106. 2011.3.

\section{Copyrights}

Copyright for this article is retained by the author(s), with first publication rights granted to the journal.

This is an open-access article distributed under the terms and conditions of the Creative Commons Attribution license (http://creativecommons.org/licenses/by/3.0/). 